\title{
Light Adaptation and Photopigment Bleaching in Cone Photoreceptors in situ in the Retina of the Turtle
}

\author{
Dwight A. Burkhardt \\ Departments of Psychology and Physiology, and Graduate Program in Neuroscience, University of Minnesota, \\ Minneapolis, Minnesota 55455
}

\begin{abstract}
Light adaptation and photopigment bleaching in cone photoreceptors were studied in the intact, superfused retina of the turtle (Pseudemys scripta elegans). A new method for measuring changes in the photopigment of cones is described. Action spectrum measurements indicate that the signals arise from the red-sensitive cones. Measurements of steady-state bleaching are well described by the monomolecular bleaching equation with a half-bleaching constant of about $5.5 \mathrm{log}$ photons $\mathrm{sec}^{-1} \mu \mathrm{m}^{-2}$.

Quantitative data on light adaptation were obtained by intracellular recording from 15 red-sensitive cones over nearly 8 decades of background illumination obtained from a helium-neon laser $(632.8 \mathrm{~nm})$. The steady-state membrane potential, $\boldsymbol{R}_{s}$, and the rate of photoisomerization of the photopigment, $\mathrm{Pi}$, rose in parallel with background illumination and then stabilized over the upper 4 decades of illumination. These results are described by the relation $\boldsymbol{R}_{s}=\boldsymbol{k} \mathrm{Pi}^{0.27}$, and suggest that about $5 \times 10^{6}$ photoisomerizations $\mathrm{sec}^{-1}$ lead to the closure of half the cone's light-sensitive channels in the steady state.
\end{abstract}

A full range of decremental and incremental flashes was used to investigate stimulus-response relations. Cones tended to generate responses of approximately constant amplitude to flashes of constant contrast over a substantial range of contrast $(\leq 3 \times)$ and background illumination ( $\sim 3-$ 4 decades). This suggests that a substantial component of contrast constancy in vertebrate vision may originate in cones. Over nearly 7 decades, the small-signal step sensitivity was found to conform closely to Weber's law (sensitivity is inversely proportional to background illumination). Thus, Weber's law extends into the ultra-high-intensity realm, some 3 decades higher than previously known for vertebrate cones.

Over the upper 3-4 decades of illumination, Weber's law behavior can be explained by the depletion of photopigment (reduced probability of the photon catch). There remains a substantial low-intensity domain for which light adaptation and Weber's law behavior are presumably mediated by other mechanisms within the cone. These might be the calcium-

\footnotetext{
Received Jan. 5, 1993; revised July 30, 1993; accepted Aug. 4, 1993.

This research was supported by a grant from the National Eye Institute (EY00406) of the National Institutes of Health. I thank Mervyn Bergman and Robert Witkofsky for technical assistance and Drs. Robert F. Miller and Ted Williams for helpful comments.

Correspondence should be addressed to Dwight $\mathrm{A}$. Burkhardt, n 218 Elliott Hall, University of Minnesota, 75 East River Road, Minneapolis, MN 55455.

Copyright (C) 1994 Society for Neuroscience 0270-6474/94/141091-15\$05.00/0
}

and/or cGMP-dependent mechanisms recently suggested by others.

[Key words: light adaptation, photopigments, photoreceptors, bleaching, Weber's law, cones, contrast]

Mechanisms of light adaptation regulate the sensitivity of the retina and are thereby instrumental for the maintenance of visual perception over the large range of ambient illumination found in natural environments. In essence, the effect of an increase in ambient illumination is offset by mechanisms that reduce the absolute sensitivity of the retina, thereby preventing overload and saturation of the response of the retinal neurons. In an ideal case, often known as Weber's law behavior, retinal sensitivity will be reduced in exact proportion to the ambient light intensity. The response of retinal neurons will then largely depend on the contrast between an object and its background, rather than the absolute light intensity of the object. Approximations toward such invariance, or contrast constancy, have been observed under various conditions in retinal neurons and human psychophysics over the years (e.g., Shapley and EnrothCugell, 1984; Burkhardt and Gottesman, 1987; Walraven et al., 1990; Whittle, 1993).

Although there is much evidence for Weber's law behavior in vertebrate vision, the retinal mechanisms for this and other aspects of light adaptation are still not fully understood. It is well established that light adaptation occurs at several stages within the retinal network, beginning in the photoreceptor cells (Shapley and Enroth-Cugell, 1984; Dowling, 1987; Fain and Matthews, 1990; Purpura et al., 1990; Walraven et al., 1990; Witkovsky and Shi, 1990). Within the photoreceptors themselves, there appear to be multiple mechanisms and several stages for light adaptation (Baylor and Hodgkin, 1974; Normann and Perlman, 1979; Valeton and van Norren, 1983; Copenhagen and Green, 1987; Fain and Matthews, 1990; Matthews et al., 1990; Schnapf et al., 1990; Dawis, 1991; Hood and Birch, 1993). It has often been proposed that depletion of photopigment by bleaching might be an important mechanism mediating light adaptation. Thus, once a large fraction of the photopigment is bleached, the effect of a further increase in the ambient light level will be exactly offset by the reduced probability of photon absorption. Bleaching could thereby account for contrast invariance and Weber's law behavior (Hecht, 1937; Boynton and Whitten, 1970; Dawis, 1978; Geisler, 1978; Walraven et al., 1990). The depletion of photopigment due to bleaching constitutes a scaling mechanism at the very first stage of the photoreceptor and thus acts in series with any mechanisms for light adaptation arising at later stages of the photoreceptor and/or retinal network. 
To define the role of bleaching in light adaptation, it would be desirable to measure directly photopigment bleaching and cone sensitivity in the same preparation under quantitatively comparable conditions. Moreover, since it is well known that both the regeneration of photopigment and metabolic integrity of the photoreceptors depend upon their normal association with the pigment epithelial cells, it is desirable to make both measurements on cones in situ in the intact, living retina. For technical reasons, such measurements will probably be very difficult to achieve in the mammalian retinas in the foreseeable future. In this paper, we report measurements on cones in the retina of the turtle that effectively meet the desired conditions. We describe a new technique in which a measuring light from a helium-neon laser is applied to the back of the eye. This technique allows us to measure bleaching and regeneration of the photopigment of red-sensitive cones in situ in the intact, superfused retina. We then report some new measurements of light adaptation and responses to positive- and negative-contrast flashes obtained by intracellular recording from red-sensitive cones. By using a helium-neon laser, we have extended the study of light adaptation to levels some 500-2000 times higher than previously investigated in vertebrate cones. Among other findings, we show that Weber's law holds almost exactly over this high-intensity range where the amount of bleaching is exceedingly high, as well as over an extensive low-intensity domain, where bleaching is negligible.

\section{Materials and Methods}

Preparation and intracellular recording. Intracellular recordings were made from cones in an eyecup-slice preparation previously described in detail (Burkhardt et al., 1989). In brief, a $2 \times 5 \mathrm{~mm}$ slice was cut through the entire posterior wall of the eye, thus keeping the sclera and pigment epithelium in place. The slice was mounted in a superfusion chamber ( $1 \mathrm{ml}$ volume) and oriented so that the layers of the retina could be viewed by infrared microscopy. The recording electrode, a glass micropipette (see below), was inserted in the retina near the inner plexiform layer and gradually advanced obliquely and downward through the retina to impale photoreceptors lying some $400 \mu \mathrm{m}$ below the cut face of the tissue (Burkhardt et al., 1989). As described in more detail below, focused light stimuli were applied to the retina in the normal physiological direction. Experiments were performed at room temperature $\left(20-23^{\circ} \mathrm{C}\right)$. The retina was superfused at $0.5-1 \mathrm{ml} / \mathrm{min}$ with a modified tissue culture medium composed of a Ringer solution (Piccolino and Gerschenfeld, 1980) supplemented with vitamins (Minimum Essential) and 11 amino acids (Eagle's Basal Medium) obtained from Sigma Chemical Co. The other components of the medium were (in mм) NaCl, $110 ; \mathrm{NaHCO}_{3}, 22 ; \mathrm{KCl}, 2.6 ; \mathrm{MgCl}_{2}, 2 ; \mathrm{CaCl}_{2}, 2$; and dextrose, 10 . The $\mathrm{pH}$ was regulated at about 7.5 by bubbling the superfusate with $98 \% \mathrm{O}_{2}$ and $2 \% \mathrm{CO}_{2}$ (Burkhardt et al., 1989).

Intracellular recordings were made with glass micropipettes filled with either $3 \mathrm{~m} \mathrm{~K}$-acetate or a mixture of $1.5 \mathrm{M} \mathrm{K}$-acetate and $1.5 \mathrm{M} \mathrm{KCl}$. No differences in the results of this report were observed due to these electrolytes, although differential effects have been found in other experimental conditions (Thoreson and Burkhardt, 1991). Electrode resistance ranged from about 400 to $900 \mathrm{M} \Omega$. Responses of cones were identified by accepted physiological criteria including acute sensitivity to the position of a moving $0.1 \times 2 \mathrm{~mm}$ slit. After a cone was impaled, a series of responses to 530 and $630 \mathrm{~nm}$ flashes were obtained to identify the spectral class. The present report is based exclusively on recordings from red-sensitive cones. The absolute flash sensitivity for a number of these cones was determined for small responses $(0.5-1 \mathrm{mV})$ evoked by $40 \mathrm{msec}, 630 \mathrm{~nm}$ flashes in fully dark-adapted retinas. The average value was $97.4 \mu \mathrm{V}$ photon ${ }^{-1} \mu \mathrm{m}^{2}(n=40, \mathrm{SD}=62.8)$, with the five most sensitive ranging from 190 to $285 \mu \mathrm{V}$ photon ${ }^{-1} \mu \mathrm{m}^{2}$. The mean maximum light-evoked response for a sample of 38 cones was $18.1 \mathrm{mV}$ $(\mathrm{SD}=4.2)$. The statistics just summarized indicate that the absolute sensitivity and maximum response of cones in the superfused eyecup slice preparation are similar to those reported previously for oxygenated eyecup preparations. Based on observations of the electroretinogram and intracellular recordings, our superfused preparations were well maintained for many hours.

Light stimulation and calibration. Three optical channels were used to stimulate the retina. Two channels were served by tungsten sources. The primary channel allowed precise spatial control of the stimulus and was used for all initial screening (see below) and other purposes. The second tungsten channel was used to provide an intense large-field adapting light in light transmission experiments (see below). The third channel, used in all experiments on light adaptation in which detailed stimulus-response data were obtained, originated from a $4 \mathrm{~mW}$ heliumneon laser $(623.8 \mathrm{~nm})$ coupled to an acousto-optic modulator (Coherent Associates model 304A). The light output of the laser was held at a steady level to light adapt the retina and then periodically increased or decreased for 400 misec to generate incremental or decremental light steps of variable magnitude. Throughout this report, the term background intensity is routinely used to refer to the steady light level to which the cone is light adapted, and the term flash intensity, to the intensity level prevailing during the flash. The flash contrast is taken as the ratio of flash to background intensity. Thus, if $I$ is the background intensity, $\Delta I$ is the incremental light step, and $F$ is the flash intensity, then flash contrast $=F / I=(I+\Delta I) / I$. Because the flash and background are spatially coincident, the contrast is purely temporal. Typically, neutral density filters were inserted in the laser beam to set the background intensity and the maximum possible incremental flash was $+3.0 \mathrm{log}$ units above the background. The intensity could be stepped as much as $-1.0 \mathrm{log}$ units below the background via the modulator. An electromagnetic shutter was closed to produce a full decrement to "complete" darkness. Calibrations showed that this amounted to a 3.0 log unit step below the background due to the presence of a small amount of stray light reaching the retina. Thus, in the figures, all data obtained for "complete" decrements are plotted $3 \mathrm{log}$ units below the background intensity. To generate extremely high levels of background illumination (>7.5 log photons $\mathrm{sec}^{-1} \mu \mathrm{m}^{-2}$ ), the steady output of the laser was increased and the maximum possible incremental flash was correspondingly limited.

The laser beam was focused to a Gaussian half-width of approximately $150 \mu \mathrm{m}$ on the retina, as measured by scanning the spot with a $10 \mu \mathrm{m}$ aperture. Our unpublished experiments and past work (Baylor and Hodgkin, 1973) show that the spot approximates the size of the center of the receptive field of turtle cones and will be a highly effective stimulus if very precisely positioned. Hence, the position of the laser spot was always very carefully adjusted $(\sim \pm 5 \mu \mathrm{m})$ to establish the optimum stimulus position. However, slight departures from the optimum position may have occurred. Moreover, some variability in the effective intensity of the laser was also likely due to variations in adjustments of the alignment of the laser beam that were always made when setting up each preparation. To avoid uncertainties arising from both factors, the effective intensity of the laser beam was calibrated physiologically, by finding the relative flash intensities of the laser and a centered spot of $630 \mathrm{~nm}$ obtained from our primary incoherent light channel (see above) that gave equivalent responses from the cone under study. The absolute intensity of the $630 \mathrm{~nm}$ light was known from direct calibrations made with a radiometer (United Detector Technologies) placed in the plane of the retina and so the effective absolute intensity of the laser in photons $\sec ^{-1} \mu \mathrm{m}^{-2}$ could be accurately determined.

Procedure and measurements for light adaptation experiments. Stimulus-response measurements on any given background were only accepted if prior and subsequent measurements in the dark-adapted state were highly reproducible. This requirement was necessary to distinguish confidently the effects of light adaptation from spurious slow changes due to extraneous factors or cell deterioration and to ensure that steadystate light adaptation was clearly reached. Thus, as a rule, dark adaptation was monitored for 15-20 min and, in total, some 40-50 min was needed to complete a dark-light-dark sequence. The criteria for reproducibility were differences between the two flanking dark-adapted series of no more than $15 \%$ and $0.2 \log$ unit, respectively, in the cone's maximum response $\left(V_{\max }\right)$ and sensitivity. For these measurements, sensitivity was defined by the intensity of a $400 \mathrm{msec}$ flash required to evoke a half-maximal response in the fully dark-adapted retina. It was not uncommon for the maximum response to differ somewhat from one series to the next while the sensitivity remained constant, within measurement error ( $\leq 0.10 \mathrm{log}$ unit). In practice, some changes in absolute amplitude were inevitable over several hours of recording. Stimulusresponse curves were therefore normalized relative to the maximal re- 
sponse obtained in the dark-adapted state. For responses obtained on a given background, amplitude was normalized relative to the average of the maximum response obtained in the preceding and subsequent dark adaptation series.

To compare results across cones, intensities were often specified relative to a unit intensity defined as the flash intensity $(400 \mathrm{msec})$ that evoked a half-maximal response in the absence of background illumination in the fully dark-adapted retina. This unit, the semisaturation constant, is denoted as sigma, and intensity is expressed in logarithmic notation. In Results, this notation is often used to specify background intensities. Thus, a background intensity of $+1 \log$ sigma is 1 log unit above the flash intensity that evoked a half-maximal response in the fully dark-adapted retina. The average value of sigma for a sample of 15 cones was $3.98 \mathrm{log}$ photons $\sec ^{-1} \mu \mathrm{m}^{-2}(\mathrm{SD}=0.32)$. Sample sizes cited in various parts of Results differ because not all cones could be studied as completely as others. Some measurements from different cones obtained with laser or white light were combined by specifying intensities in units of sigma. In other cases, white light intensities were converted to equivalent photons $\sec ^{-1} \mu \mathrm{m}^{-2}$ at $630 \mathrm{~nm}$ by finding the relative flash intensities of white and $630 \mathrm{~nm}$ light required to evoke responses of equal amplitude (Burkhardt et al., 1988). Background or flash intensities were converted to photoisomerizations $\mathrm{sec}^{-1}$ for redsensitive cones, by using the factors given by Baylor and Fettiplace (1975) for the collecting area of the inner segment, the transmission factor from inner to outer segment, the fraction of light absorbed by the photopigment, and the quantum efficiency of bleaching. As described in more detail in Discussion, the fraction of light absorbed decreases when steady background illumination depletes the cone photopigment.

Equivalent luminance of background illumination. Because the background lights used in the present report are the most intense so far used in studies of vertebrate cones, it is of interest to know what luminance levels of objects in the natural environment might lead to such high levels of retinal illumination. Rather than make uncertain assumptions about the optical constants, preretinal transmission, and spectral sensitivity factors of the turtle eye, the following measurements and procedures were made to equate the background illumination used in the present experiments to the luminance of objects in the external environment. (1) A photometer was used to measure the luminance of a large screen illuminated with white light. (2) An aperture about $3 \mathrm{~mm}$ in diameter was cut in the back of a turtle eye and the eye was positioned to be looking at the screen from a distance of about $3 \mathrm{~m}$. (3) A fiber optic of $2 \mathrm{~mm}$ diameter was then inserted in the plane of the retina and the irradiance measured with a radiometer (United Detector Technology model 350). (4) The fiber optic was subsequently placed in the plane normally occupied by the eyecup slice preparation, illuminated by white light obtained from channel $I$ of the stimulator, and a second measurement taken with the radiometer. (5) The object luminance equivalent to a given retinal irradiance (photons $\sec ^{-1} \mu \mathrm{m}^{-2}$ ) for the redsensitive cones was then calculated from the measurements in steps 1 , 3 , and 4 above, along with the white- $630 \mathrm{~nm}$ equivalence calibrations described in the previous paragraph.

Light transmission measurements. The back of the intact eye was cleaned by trimming away the extraocular muscles, optic nerve, and loose tissue. A sharp hypodermic needle was then used to produce an L-shaped score in the opaque sclera tunic that covers the cartilaginous sclera proper. The sclera proper is transparent in the turtle and many other lower vertebrates (Walls, 1963). With care, the tunic could be scored without disrupting the sclera proper or puncturing the eye. The tip of the needle was then used to peel the tunic gradually away from the sclera, starting at the intersection of the legs of the $L$ and continuing until about $4 \mathrm{~mm}^{2}$ of sclera was exposed. When the tissue was kept moist with a drop of Ringer's and viewed under an ordinary dissecting microscope, the tunic was readily distinguished from the sclera proper. Remaining remnants of the tunic were removed with the needle or fine emery paper. In all cases, the exposed area was somewhat ventral of the posterior pole of the eye so that the region of the optic nerve and the sclera pits associated with the extra ocular insertions were avoided. The optic nerve head was painted with black waterproof ink to prevent stray light from passing through the optic nerve and reaching the photodiode (see below). The eye, intact up to this stage, was now hemisected, the anterior portion discarded, and most of the vitreous humor removed with small pieces of absorbent paper. The resulting eyecup was then placed in a small chamber for light transmission measurements and continuously superfused in a manner identical to that described above under intracellular recording.
Figure 1 lays out the essential elements of the measuring system. Light $(632.8 \mathrm{~nm})$ from the laser/modulator system described above, was modulated in square wave fashion at $18 \mathrm{~Hz}$ and directed into a $2 \mathrm{~mm}$ solidcore fiber optic (labeled F1). On other occasions, the laser beam was extinguished and the output of an infrared emitting diode (maximum emission at $880 \mathrm{~nm}$ ), also modulated at $18 \mathrm{~Hz}$, was directed upon F1. Most of the light leaving F1 entered fiber optic F3 while about $10 \%$ was reflected by a beam splitter $(\mathrm{Sp})$ to enter fiber optic F2. Fiber optic F3 carried light into the chamber housing the eyecup. The eyecup was aligned so that the end of F3 ( $2 \mathrm{~mm}$ in diameter) was approximately centered and nearly abutted the exposed area of sclera (S). The beam exiting $F 3$ was incident upon the sclera at an intensity of approximately $6.5 \mathrm{log}$ photons $\mathrm{sec}^{-1} \mu \mathrm{m}^{-2}$. It then passed through the transparent sclera and into the highly absorbent pigment epithelial layer (PE). A very small but measurable fraction of this light was not absorbed by the pigment epithelial layer, traversed the retina (R), and was collected and converged by two lenses ( $\mathrm{Ll}$ and $\mathrm{L} 2$ ) so that an image of the retinal surface was formed in the plane of the aperture (Ap). When setting up a preparation, the laser was temporarily set at maximum intensity and the plane of Ap viewed with an auxiliary eyepiece (not shown). Usually, the patch of transmitted light was just detectable. The aperture, mounted on a micromanipulator, was then centered on the patch and stopped down to slightly less than the size of the patch, in an effort to exclude stray light. The light passing through Ap fell upon an electromagnetic shutter (Sh) that could be opened to permit light to enter a $3-\mathrm{mm}$ diameter fiber optic (F4), which led to a photodiode/operational amplifier module $(\mathrm{P})$. The output signal was fed to a narrow band-pass filter centered at $18 \mathrm{~Hz}$ with upper and lower $-3 \mathrm{~dB}$ frequencies of about 18.5 and $17.5 \mathrm{~Hz}$, respectively. The resulting $18 \mathrm{~Hz}$ sine wave was displayed on an oscilloscope and sent to a computer that measured the average amplitude of the signal for a sample of 45 cycles $(2.5 \mathrm{sec})$. This signal was then scaled relative to that generated by applying an infrared beam of fixed intensity at $F 1$ in the absence of the laser beam, on the assumption, as in the method of reflection densitometry (Rushton and Henry, 1968; Alpern, 1971; Rushton, 1972; van Norren and van der Kraats, 1981), that the infrared is unaffected by changes in the photopigment and may be used to factor out changes in the laser signal unrelated to the effects of the photopigment. To factor out possible errors due to drift in the signal sources, both the laser and infrared transmitted signals were also scaled relative to their respective reference signals obtained from the light reflected from Sp into F2. After the initial alignment procedure discussed above, the intensity of the input laser beam was reduced, typically about $1 \log$ unit, so that the measuring beam was as weak as possible while still yielding a satisfactory signal: noise ratio. At this level of intensity, the measuring laser light was invisible when using the inspection system described above and when turned on, it never evoked a detectable electroretinogram. In auxiliary experiments with normal retinal illumination, it was found that weak lights that produced no detectable bleaching evoked a clearly detectable electroretinogram. It may thus be concluded that the measuring beam used in the present method was too weak to produce measurable bleaching.

The present method assumes that the photopigments are the only elements in the preparation that undergo substantial changes in $a b$ sorption as a result of exposing the retina to an adapting light. Changes in light transmission may thus be used to monitor changes in the photopigment concentration by computing the ratio, $R$, defined as

$$
R=L / D,
$$

where $L$ and $D$ are the amplitudes, scaled as described above, of the photodiode signals obtained, respectively, after the retina had been adapted to a given adapting light or fully dark adapted. In practice, the value of $D$ used in Equation I was taken as the average of the steadystate signals measured before and after the retina was exposed to each adapting intensity. This procedure was found essential to minimize the effects of long-term drift in the measurements but made the experiments very time consuming since the preparation was always dark adapted for 15-20 min before and after each adapting intensity studied. To measure $L$, the adapting light was extinguished for $4 \mathrm{sec}$ and after a $1.1 \mathrm{sec}$ delay, a $2.5 \mathrm{sec}$ sample of the transmitted signal was obtained. These measurements were typically made every $20 \mathrm{sec}$ and thus, for quantitative analyses, the effective adapting intensity was taken as $16 / 20$ of the nominal value. After exposure to a number of adapting intensities, including those producing full bleaching, the fraction of pigment bleached $(B)$ by a given adapting light was calculated: 


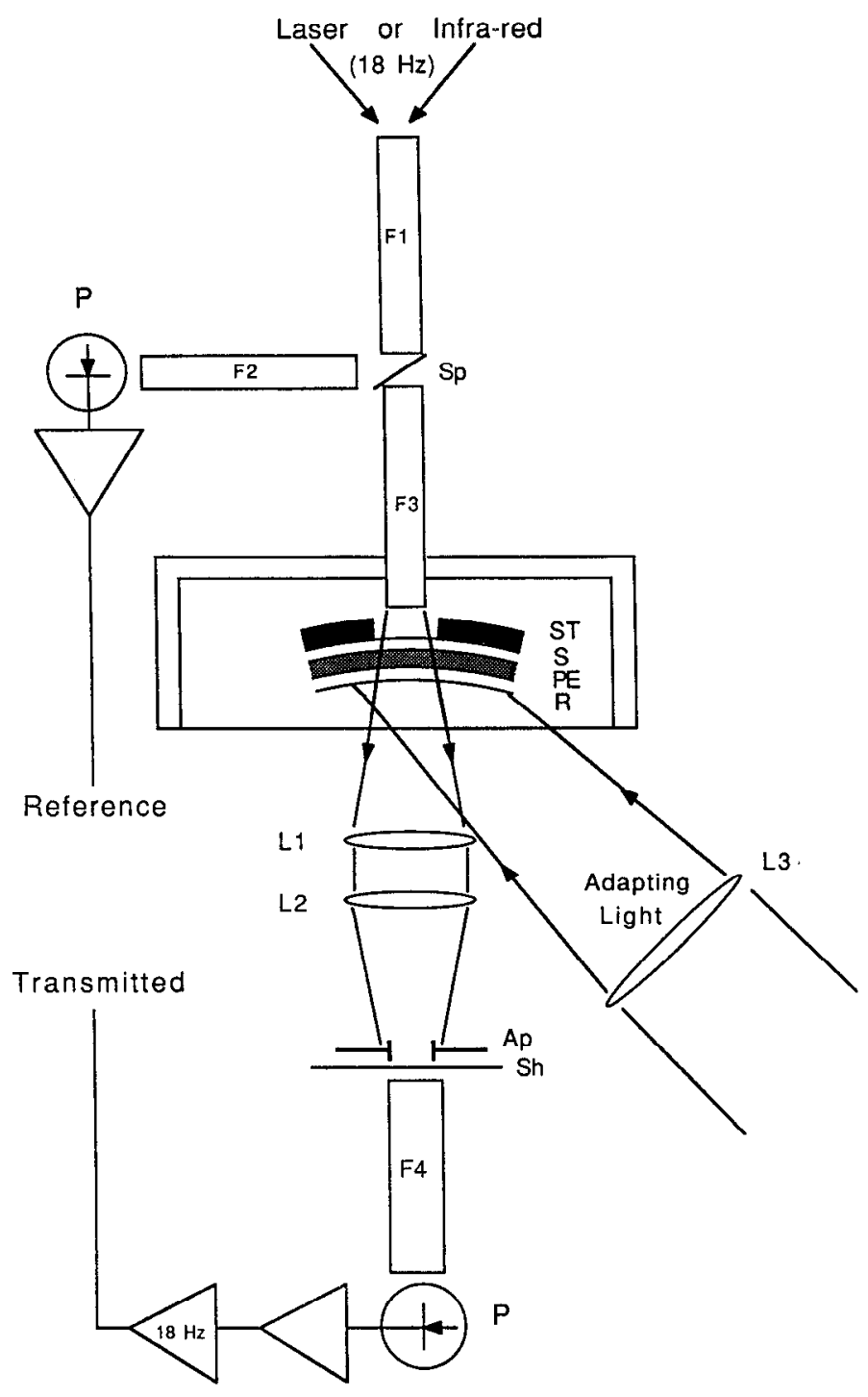

Figure 1. System for measuring light transmission through the retina. $S T$, scleral tunic; $S$, sclera proper; $P E$, pigment epithelium; $R$, retina; $P$, photiodide; $F 1-F 4$, fiber optic light guides; $A p$, aperture; $S h$, shutter; $L 1-L 3$, lenses; $S p$, beam splitter. See Materials and Methods for details.

$$
B=(R-1) /\left(R_{\max }-1\right),
$$

where $R_{\max }$ is the value of $R$ obtained on the most intense adapting field (see below). The proportion of photopigment present, $P$, is then given by $(1-B)$.

In practice, the measured value of $R$ will depend on the fraction of the measuring light that passes through the photoreceptors relative to that which reaches the photodiode without passing through the photoreceptors. The latter fraction, called the stray light, will act to reduce the measured changes due to the photopigment, much as previously analyzed for the method of retinal densitometry (Ripps and Weale, 1965; Smith et al., 1983; Baker et al., 1991). Thus, if $S$ represents the signal generated by the stray light component and $C_{L}$ and $C_{D}$ are, respectively, the signals generated by the light that passes through the cone photoreceptors in the light and dark, Equation 1 may be written as

$$
R=\left(C_{L}+S\right) /\left(C_{D}+S\right)
$$

Since red-sensitive cones in the turtle in the fully dark-adapted state absorb about $36 \%$ of the incident light (Baylor and Fettiplace, 1975), the maximum possible value of $R$ should be about 1.56 if there were no stray light. On a verage, the observed maximum value of $R$ was about 1.05. It thus follows from Equation 3 that the stray light component was about six times greater than the component passing through the cones. This is not surprising since the cone outer segments in the turtle are small $(\sim 1 \mu \mathrm{m}$ diameter) and, when viewed from behind, would occupy only a fraction of the total surface area receiving the incident light. Furthermore, some stray light was almost certainly reflected off the back of the preparation and off surfaces in the chamber to find its way to the measuring photodiode. The eyecup was surrounded with black Vaseline to minimize stray light escaping around the edge of the eyecup. (It is believed that stray light due to spurious reflections accounted for our failure to detect photopigment bleaching in preliminary experiments in which we attempted to apply the method of reflection densitometry to the turtle eyecup.) Although the presence of stray light prevents the transmission method from yielding valid estimates of the photopigment density, even when $S$ is the larger term in Equation 3, a valid index of the relative amount of bleached pigment will still be extracted from Equation 2.

The adapting light originated from a tungsten source. Before reaching the focusing lens, L3, shown in Figure 1, the light passed through a heat filter, about $45 \mathrm{~cm}$ of a fiber optic, an aperture, a collimating lens, and neutral density and/or interference filters ( $10 \mathrm{~nm}$ half-band pass). The adapting field was about $5 \mathrm{~mm}$ in diameter on the retina and always covered the area of the measuring beam. Preliminary experiments showed that the upper limit of steady-state bleaching was reached when the retina was exposed to the maximum intensity of white light available from this system (equivalent to $7.90 \mathrm{log}$ photons $\sec ^{-1} \mu \mathrm{m}^{-2}$ at 630 $\mathrm{nm})$. This intense white light was therefore used in all preparations to establish the upper limit of bleaching, whereas all other adapting intensities were obtained with an interference filter (typically $630 \mathrm{~nm}$ ) in the adapting beam. The adapting light was obliquely incident upon the retina (Fig. 1). The bleaching of human cone photopigments (Coble and Rushton, 1971) and the excitation of turtle cones (Baylor and Fettiplace, 1975) are known to be directionally sensitive. The following procedure was therefore taken to calibrate the effective intensity of the oblique adapting light. Intracellular recordings were made from red-sensitive cones to find the physiologically equivalent intensity levels of the adapting channel (when presented as $630 \mathrm{~nm}$ flashes) and our primary light channel. Since the primary light channel illuminated the cones in the normal, axial direction and was accurately calibrated (see above), the effective intensity of our adapting lights could be determined.

\section{Results}

Measurements of photopigment bleaching

Basic properties of light transmission signals

The upper trace of Figure 2 shows a record of the filtered photodiode signal generated by light that passed through the transparent layer of the sclera, the pigment epithelium, and the neuroretina. The lower trace shows the reference signal. These traces suggest that the signal : noise is good enough that measurements of the signal amplitude may be capable of resolving small differences in light transmission. Figure 3 shows transmission measurements made (1) in the dark-adapted retina, (2) while the retina was illuminated by a steady adapting light of $630 \mathrm{~nm}$ at an intensity of $6.63 \mathrm{log}$ photons $\mathrm{sec}^{-1} \mu \mathrm{m}^{-2}$ for $5.3 \mathrm{~min}$, and (3) over a period of some 20 min after the adapting light was extinguished and the retina allowed to dark adapt. The ordinate is the scaled photodiode signal as given by Equation 1. The photodiode signal, and thus light transmission, increases while the adapting light is on. This is the direction of change required if the bleaching of photopigment is being measured. Bleaching of photopigment in normal cones in situ is fully reversed by dark adaptation. Figure 3 shows that analogous reversibility is found in our measurements of light transmission. After the background illumination is extinguished, transmission gradually declines and, after about $15 \mathrm{~min}$, returns to a value similar to the original, dark-adapted level. The time for the light transmission to return to the dark level ranged from about 2 to $15 \mathrm{~min}$, depending on the intensity of the adapting light. A similar range of recovery times was observed for cone dark adaptation in the intracellular recording experiments (see Materials and Meth- 


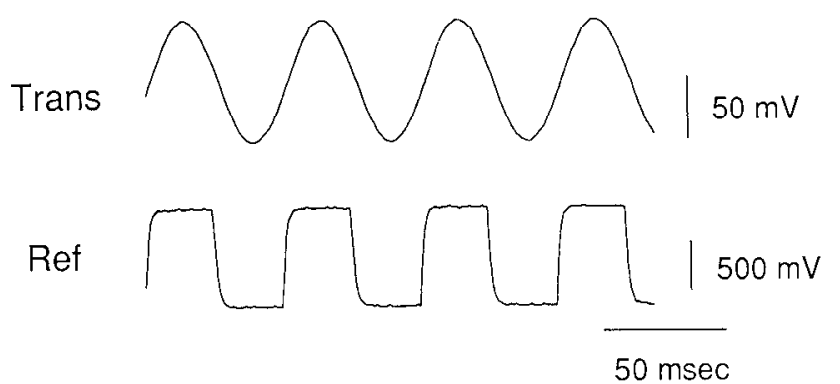

Figure 2. Photodiode signals for light transmitted through the retina (Trans) and for the reference light (Ref).

ods), with at least $15 \mathrm{~min}$ being required for full recovery after exposure to very intense adapting fields. Although our measurements are as yet too limited to permit quantitative conclusions about kinetics, it is apparent that the light transmission signals behave as expected for visual pigments (Dartnall, 1962; Rushton and Henry, 1968; Hollins and Alpern, 1973; Bonds and MacLeod, 1974). Thus, the initial rate of bleaching was much higher for intense adapting fields, whereas recovery to the dark level occurred more quickly after exposure to weaker adapting lights.

\section{Measurements in the steady state}

The effect of steady background illumination on steady-state transmission was measured as described in detail in Materials and Methods. Figure 4 shows results from two preparations. Each set of measurements took some $3 \mathrm{hr}$, since pains were taken to ensure that steady-state conditions were reached (see Materials and Methods). The results are similar for both preparations. The ordinate shows the valuc of $P$, the fraction of pigment present in the unbleached state (see Materials and Methods). The smooth curve represents the equation

$$
P=I_{h} /\left(I_{h}+I\right),
$$

where $I$ is the intensity of the steady background illumination in photons $\sec ^{-1} \mu \mathrm{m}^{-2}, P$ is as defined above, and $I_{h}$ is the intensity that reduces $P$ to 0.5 , that is, the half-bleaching constant. This equation has been shown to fit the steady-state bleaching data for human cones measured by reflection densitometry (Rushton and Henry, 1968; Rushton, 1972; Hollins and Alpern, 1973). The half-bleaching constant for the bestfitting smooth curve in Figure 4 is $5.57 \log$ photons $\mathrm{sec}^{-1} \mu \mathrm{m}^{-2}$.

Steady-state bleaching measurements were carried out in a total of six preparations. The half-bleaching constant agreed well across preparations (mean $=5.54 \log$ photons $\sec ^{-1} \mu \mathrm{m}^{-2}, \mathrm{SD}$ $= \pm 0.13, n=6)$ and the bleaching equation gave a good fit across the sample (mean $r^{2}=0.975, \mathrm{SD}= \pm 0.015, n=6$ ).

\section{Action spectrum for bleaching}

The half-bleaching constant given above applies for $630 \mathrm{~nm}$, the wavelength of the adapting field used in the experiments described to this point. Measurements of the action spectra of turtle photoreceptors (Baylor and IIodgkin, 1973) suggest that our measuring wavelength of $632.8 \mathrm{~nm}$ will be absorbed about 25 times more strongly by red-sensitive cones than by greensensitive cones or the rods and it is also known that the redsensitive cones constitute some $80 \%$ of the total photoreceptor population (Ohtsuka, 1985). It would thus seem likely that our transmission measurements largely arise from the red-sensitive

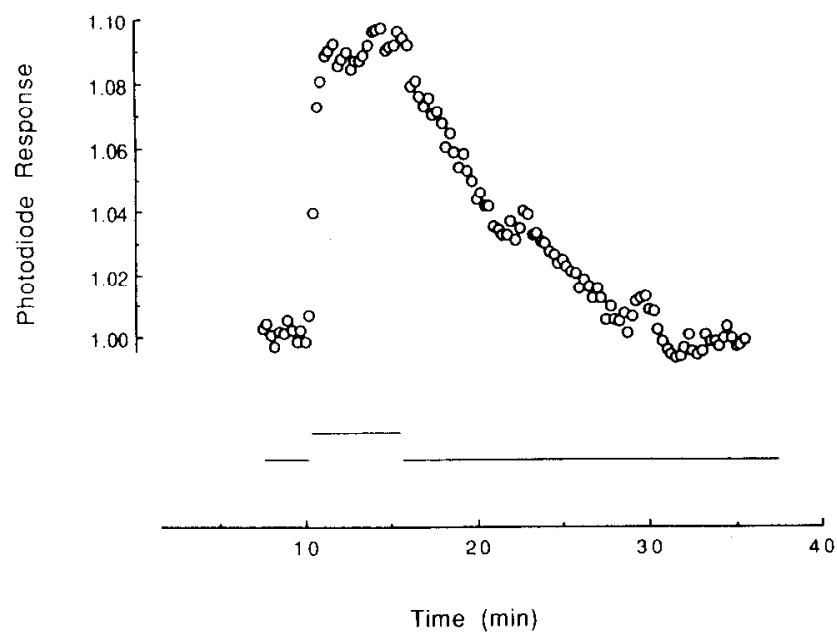

Figure 3. Change in photodiode response to transmitted light during and after the retina was exposed for $5.3 \mathrm{~min}$ to an intense adapting light of $6.63 \log$ photons $\mathrm{sec}^{-1} \mu \mathrm{m}^{-2}$ at $630 \mathrm{~nm}$.

cones. If so, the efficiency of bleaching at wavelengths other than 630 should be predicted from the action spectra of the redsensitive cones. Figure 5 summarizes the results of five experiments that were designed to compare the half-bleaching constants for adapting lights of $630 \mathrm{~nm}, 530 \mathrm{~nm}$, and $700 \mathrm{~nm}$. The ordinate is the reciprocal of the steady photon levels required to produce a value of $P=0.5$. The solid circles show the mean measurements normalized relative to $630 \mathrm{~nm}$.

There are four types of red-sensitive cones in the turtle retina that have different spectral properties due to differences in their oil droplets. Since our measuring light covers a large population of cones, the predicted efficiency of bleaching must take into account the effective absorption spectra and relative incidence of all four cone types. The predicted efficiency of bleaching was calculated from the data of Ohtsuka (1985) as follows. Estimates of sensitivity relative to $630 \mathrm{~nm}$ were taken from the average spectral sensitivity measured at 530,630 , and $700 \mathrm{~nm}$ for each of the four cone types (Ohtsuka, 1985, his Fig. 2). The sensitivities were converted to linear values, $s$, and weighted by the incidence, $i$, of the cone type. The resultant predicted sensitivity,

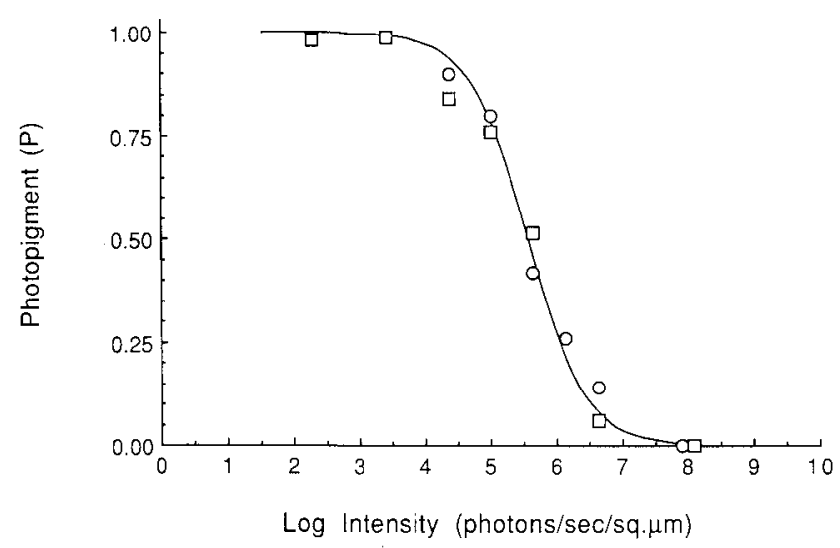

Figure 4. Fraction of photopigment unbleached, $P$, as a function of the intensity of steady adapting lights of $630 \mathrm{~nm}$ in steady-state, equilibrium conditions. Symhols show results from two cones. The smooth curve represents the bleaching equation (Eq. 4) with the half-bleaching constant set at $5.57 \mathrm{log}$ photons $\mathrm{sec}^{-1} \mu \mathrm{m}^{-2}$. 


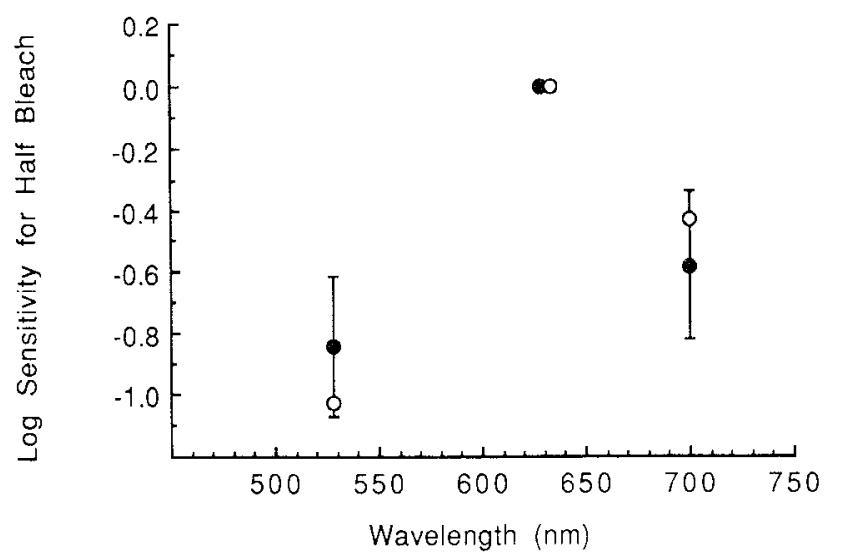

Figure 5. Action spectrum for half-bleaching. Solid circles are from transmission measurements. Open circles are results predicted if the transmission signal arises exclusively from red-sensitive cones. See Results.

$S$, at each wavelength was then computed:

$$
S=\sum_{n=1}^{4}\left(s_{n} \cdot i_{n}\right) \text {. }
$$

The computed values, normalized relative to sensitivity at 630 $\mathrm{nm}$ and converted to logarithmic units, are shown by the open circles in Figure 5. The measured and predicted values are in good agreement. This strongly supports the conclusion that our transmission method is selectively measuring the photopigment of the red-sensitive cones.

It might be supposed that green-sensitive cones and/or rods could make significant contributions to the transmission measurements when the concentration of the red-sensitive photopigment has been substantially reduced by the use of intense adapting lights. This situation is analyzed in detail in the Appendix. It is shown that the combined contributions from greensensitive cones and rods would be expected to increase the measured half-bleaching constant by only about $0.11 \log$ unit for the data of Figure 4, a factor within the experimental measurement error (SD - \pm 0.13 ). The analysis also indicates that the results of Figures 3 and 5 should not be seriously distorted by contributions from green-sensitive cones and rods.

\section{Light adaptation in cones}

\section{Observations from a very stable recording}

Quantitative data on light adaptation were obtained from a sample of 15 red-sensitive cones, following the protocols given in Materials and Methods. Recordings from several cones were held for at least $2 \mathrm{hr}$. In the best case, hereafter called "cone A," the recording was held for more than $3 \mathrm{hr}$ and the signal: noise was unusually good. Figure $6 \mathrm{~A}$ shows superimposed responses of this cone evoked by $400 \mathrm{msec}$ flashes of light in the fully dark-adapted retina. The hyperpolarizing responses range up to about $24 \mathrm{mV}$ and are similar in waveform to those observed previously by others. After the responses of Figure $6 \mathrm{~A}$ were recorded, the conc was exposed to a steady background light at an intensity of $1.65 \mathrm{log}$ sigma (equivalent to $5.65 \mathrm{log}$ photons $\sec ^{-1} \mu \mathrm{m}^{-2}$ ). The course of light adaptation was followed by monitoring the increase of the response evoked by a full decremental flash. The response increased over time, while the resting membrane potential gradually depolarized to a steady level. As a rule, such recovery was largely complete in 2-3 min but we routinely waited at least $5 \mathrm{~min}$, and $10-15 \mathrm{~min}$ in the case of very intense backgrounds, to ensure that steady-state light adaptation was unequivocally reached.

In Figure $6 B$, the cone had reached the steady state and the superimposed traces show responses evoked by a series of incremental flashes of variable magnitude. The maximum hyperpolarization evoked by a saturating flash in Figure $6 B$ is somewhat less than that evoked by a saturating flash in the dark in Figure $6 A$. Figure $6 C$ shows superimposed responses evoked by a series of decremental flashes of variable magnitude. The largest response, evoked by a full decrement, that is, a $400 \mathrm{msec}$ step to complete darkness, overshoots the dark membrane potential level (shown as a dash) by some $6 \mathrm{mV}$. After the termination of the step, the responses to the three largest decrements show a clear hyperpolarizing overshoot. After obtaining the responses of Figure $6 C$, the background was turned off and the cone allowed to dark adapt for some $15 \mathrm{~min}$ until the responses to a weak test flash were judged to be constant. The series of responses in Figure $6 D$ was then obtained in response to light flashes of variable intensity. This response series was virtually identical to that of Figure $6 \mathrm{~A}$ recorded some $25 \mathrm{~min}$ earlier.

Figure $6 E-H$ shows responses recorded in the steady state on backgrounds some $250 \times$ dimmer and $200 \times$ more intense, respectively, than that used in $B$ and $C$. Figure $6, E$ and $F$, shows responses on a background whose intensity ( $3.25 \mathrm{log}$ photons $\mathrm{sec}^{-1} \mu \mathrm{m}^{-2}$ ) was equivalent to $-0.75 \mathrm{log}$ sigma. The response range for increments is much larger than that for decrements. The upper two decremental responses show a slight depolarizing overshoot above the dark membrane potential (marked by the dash). Figure $6, G$ and $H$, shows responses evoked on a background of $3.95 \mathrm{log}$ sigma (7.95 log photons $\left.\sec ^{-1} \mu \mathrm{m}^{-2}\right)$. In contrast to the responses in $E$ and $F$, the response range for decrements is larger than that for increments and both response sets show relatively complex waveforms with prominent overshoots at stimulus offset.

\section{Measurements of the steady-state membrane potential}

The resting membrane potential in the dark-adapted state is shown by the horizontal lines in Figure 6 . The average value over the course of the recording was about $-33 \mathrm{mV}$. The second largest response in Figure $6 A$ was evoked by a flash of $1.65 \mathrm{log}$ sigma applied in the dark. When the same light was applied continuously, the hyperpolarization gradually depolarized to reach the steady-state level shown by the baseline in Figure $6 B$. The tendency of the membrane potential to depolarize toward the dark level in the presence of steady illumination has been widely observed in various species and is generally believed to be a fundamental manifestation of light adaptation. Figure 7 gives a quantitative view of this behavior in our sample of turtle cones. The dashed curve, based on average results in 23 cones (see below), represents the peak hyperpolarization evoked when a background light is turned on and the retina is fully dark adapted. In contrast, the circles show measurements of the magnitude of the steady-state hyperpolarization observed after background light has been maintained for some $10 \mathrm{~min}$. The solid circles show the average steady-state results collected from a sample of 15 cones, and the open circles show results for cone A of Figure 6 . The results of Figure 7 agree with previous observations that the stimulus-response curve becomes shallower after 1-2 sec of maintained illumination (Baylor and Hodgkin, 1974) and the membrane potential subsequently depolarizes to reach a steady-state value about half that evoked in the dark 
A

DA
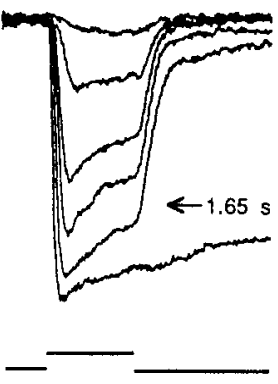

$E$

$-0.75 s$

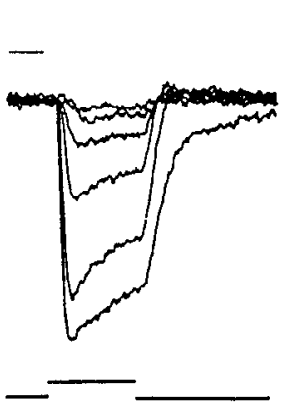

B

$1.65 \mathrm{~s}$
C

$1.65 \mathrm{~s}$
$D$

D A
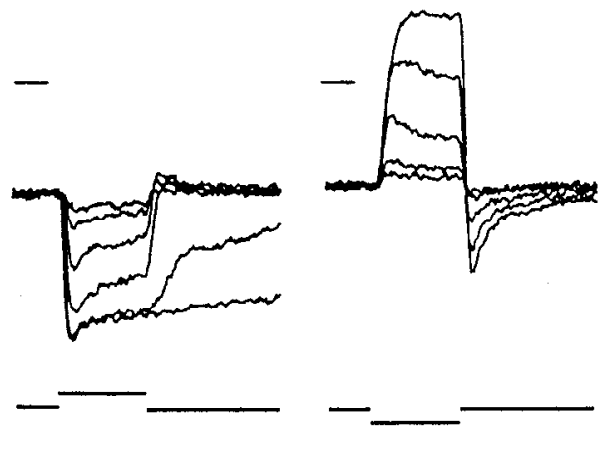

G

$3.95 \mathrm{~s}$
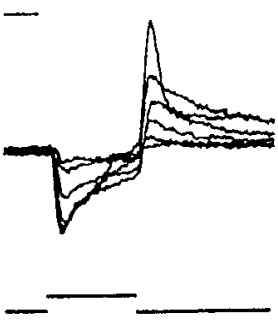

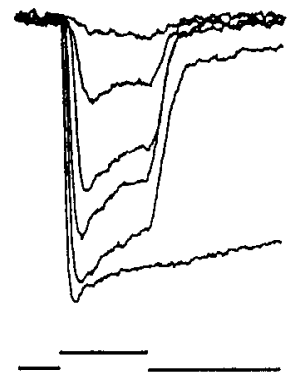

$5 \mathrm{mV}$

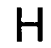

$3.95 \mathrm{~s}$

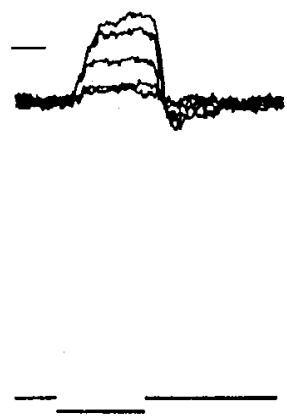

Figure 6. Responses of a cone to flashes in the dark ( $A$ and $D$ ) and in the presence of steady background illumination $(B, C, E-H)$. The intensity of the background illumination is given by the number above each set of recordings in logarithmic units relative to the intensity, sigma, that produces a half-maximal response in the dark ( sigma $=4.0$ $\log$ photons $\mathrm{sec}^{-1} \mu \mathrm{m}^{-2}$ ). A horizontal line shows the level of the resting potential in the dark (approximately -33 $\mathrm{mV}$ ). The flash intensities for the responses in $A$ and $D$ are equal to -1.75 , $-0.75,0.25,0.75,1.65$, and $3.95 \mathrm{log}$ sigma. The responses in $C, F$, and $H$ were generated by a series of negative steps of $0.09,0.18,0.47,0.90$, and 3.0 $\log$ units relative to the background. The responses in $B, E$, and $H$ were generated by a series of positive steps of 0.09 , $0.20,0.50,0.98,1.96$, and $2.92 \log$ units relative to the background.
(Normann and Perlman, 1979). Figure 7 extends measurements to intensities some $2 \log$ units higher than previously studied and thereby shows that the potential stabilizes at about $0.5 V_{\max }$ over a range of high background intensity of at least $4 \log$ units (2-6 log sigma or equivalently, about 6-10 log photons $\mathrm{sec}^{-1}$ $\mu \mathrm{m}^{-2}$ ). Over this range, the mean steady-state potential was 0.49 $V_{\max }(\mathrm{SD}= \pm 0.05, n=37)$. Light flashes on background illu- mination evoke changes in voltage from the steady-state levels shown in Figure 7. The stabilization of the membrane potential at high background intensities might preserve about one-half of the response range for positive and negative contrast steps, respectively. To consider this and other aspects of light adaptation, we now concentrate exclusively on the changes in voltage evoked by flashes of variable magnitude and polarity.

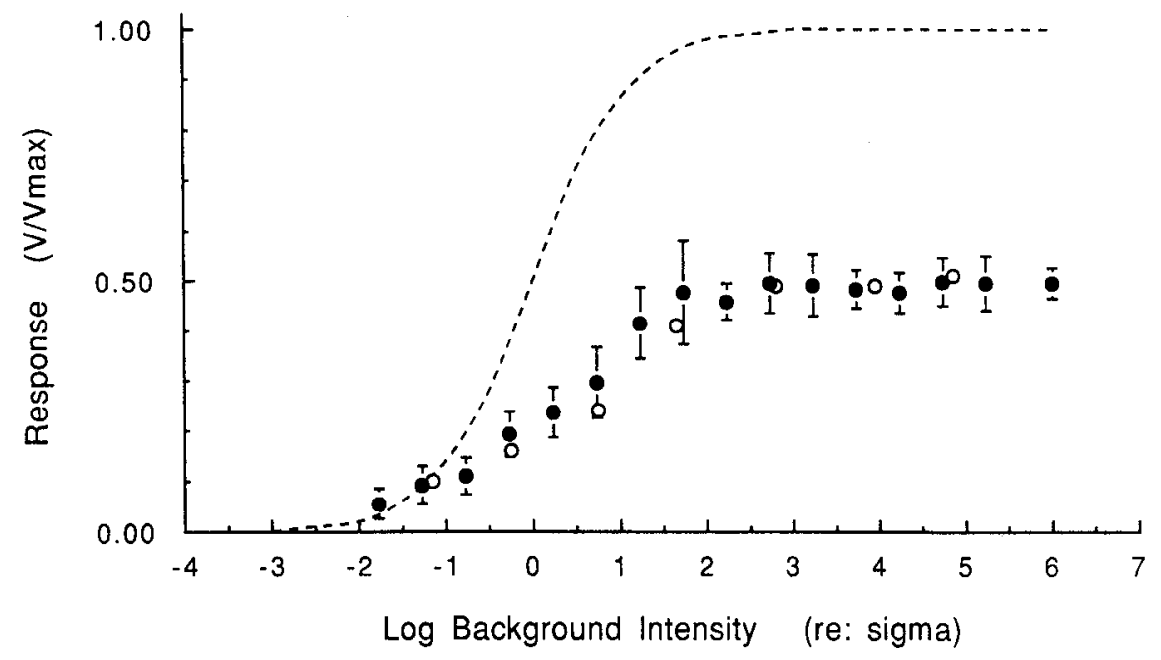

Figure 7. Steady-state potential maintained in steady background illumination for the cone of Figure 6 (open circles) and for results collected from a sample of 15 cones (solid circles). The potential is expressed as a fraction of the maximum hyperpolarization evoked by a saturating flash in the dark $\left(V_{\max }\right)$. The smooth curve represents the peak hyperpolarization evoked when a background light is first turned on in the dark. $\log$ intensity of 0 sigma $=4.00$ and 4.01 log photons $\mathrm{sec}^{-1} \mu \mathrm{m}^{-2}$, respectively, for the data plotted as solid and open symbols. 


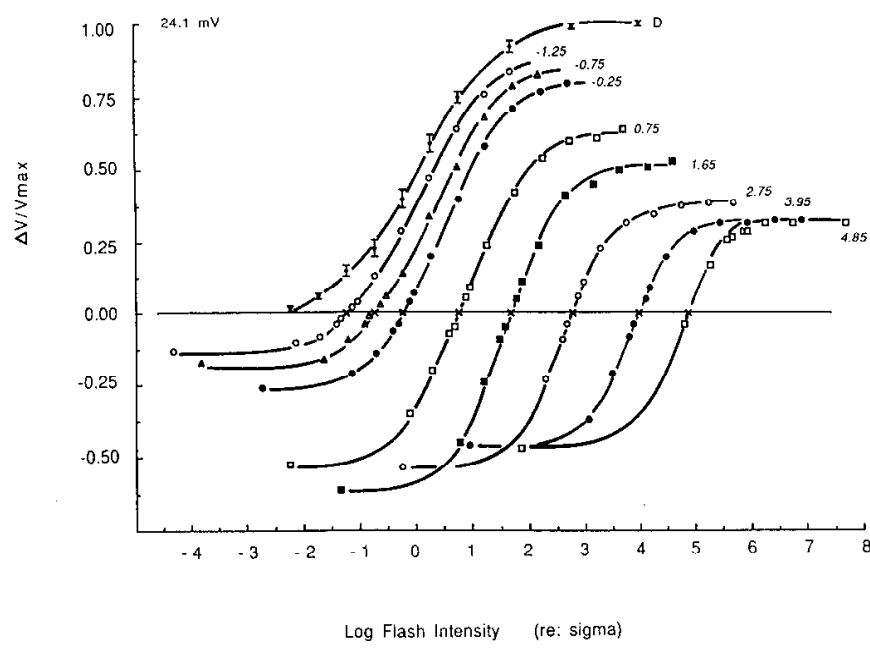

Figure 8. Stimulus-response relations for flashes applied in the dark (curve $D$ ) or on steady background illumination. Responses evoked by increments and decrements are given positive and negative signs, respectively, and scaled relative to the maximum hyperpolarizing response evoked in the dark $\left(V_{\max }\right)$. The average value of $V_{\max }$ over the entire recording period was $24.1 \mathrm{mV}$, as shown at the upper left. The number to the right of each curve indicates the background intensity in units of log sigma. The steady-state response to these backgrounds is shown by the open circles in Figure 7. The backgrounds and specific values for the steady-state response are $D, 0.0 ;-1.25,0.10 ;-0.25$, $0.16 ; 0.75,0.24 ; 1.65,0.41 ; 2.75,0.49 ; 3.95,0.49 ;$ and $4.85,0.51$. Smooth curves were drawn by eye. The recording was lost before the decremental series could be completed on the background 4.85 sigma. See Results for details.

\section{Stimulus-response curves}

Figure 8 summarizes measurements obtained from cone $\mathrm{A}$ on eight background levels, following the protocol outlined in Materials and Methods. The vertical axis shows the peak amplitude of the changes in voltage evoked by positive and negative steps. Amplitude is normalized relative to the maximum hyperpolarizing response evoked in the dark-adapted state (see Materials and Methods). Symbols above and below zero represent hypcrpolarizing and depolarizing responses evoked by positive and negative steps, respectively. $\mathrm{A} \times$ marks the background intensity. All the smooth curves in Figure 8 are drawn by eye. Curve $\mathrm{D}$ at the far left is drawn through the average of eight sets of interspersed control measurements taken over some $3 \mathrm{hr}$ when the retina was periodically returned to the fully dark-adapted state. The standard errors are small, showing that the darkadapted sensitivity and stimulus-response curve were highly reproducible throughout the course of the recording.

Figure 8 illustrates two characteristic effects of light adaptation on the stimulus-response curves that were observed throughout our sample of cones. (1) The total response range (i.e., the total excursion in voltage between the maximum depolarizing and hyperpolarizing responses) was not invariant with light adaptation. With respect to that found in the dark, the total response range often increased to $110-120 \%$ on moderate backgrounds and then invariably decreased to some $60-80 \%$ on very intense backgrounds. (2) The response evoked by a full decremental flash (i.e., a step to complete darkness) varied with background level, reaching a peak at an intermediate intensity and then decreasing on higher background intensities. This effect is clearly illustrated in Figure 8. In a sample of 19 cones, the maximum response range for decrements was found, on average, on a background of $1.77 \log$ sigma $(\mathrm{SD}= \pm 0.50)$. The expansion of the response to decrements seems partly due to the emergence of a depolarizing overshoot above the dark membrane potential level, as illustrated in Figure $6 C$. In a sample of 15 cones, the maximum overshoot was $+3.9 \mathrm{mV}(\mathrm{SD}= \pm 1.95)$ relative to the dark-adapted membrane potential and occurred on a background intensity of $0.95 \mathrm{log}$ sigma $(\mathrm{SD}= \pm 0.56)$.

As a rule, dark-adapted stimulus-response data were reasonably well described by the modified Michaelis-Menton equation, $V / V_{\max }=I^{n} / I^{n}+s^{n}$, where $I$ is the flash intensity, $V$ and $V_{\max }$ are the observed and maximum peak response, respectively, and $s$ is the intensity (sigma) that evokes a half-maximal response. For 23 cones, the average value of $n$ was 0.80 (SD $=$ \pm 0.08 ), and thus somewhat less than the value of 1 required by the Michaelis-Menton relation, and found to hold reasonably well in eyecup preparations under most conditions (Baylor and Hodgkin, 1973, 1974; Normann and Pcrlman, 1979; Pulvinage and Green, 1990). Statistical analysis indicated that the value of the experiment, $n$, was not significantly dependent on flash duration ( 40 vs $400 \mathrm{msec}$ ), the use of a small spot versus large field stimuli ( $150 \mu \mathrm{m}$ vs $2.2 \mathrm{~mm}$ ) or laser versus white light. In view of the relatively complex and variable effects of background illumination described above (Figs. 6,8), no attempt was made to find a general mathematical description for the form and variation of the stimulus-response curves as a function of background intensity.

\section{Effects of flash contrast}

Flashes of equal contrast evoke equal-amplitude responses independent of the background intensity wherever the curves in Figure 8 stay parallel to one another. Contrast constancy of this sort holds rather well over an appreciable range in Figure 8 . This is highlighted in Figure 9, where responses to flashes of fixed contrast are shown on three different background levels that cover an intensity range of 50,100 (from -0.75 to $3.95 \mathrm{log}$ sigma). Contrast is specified as the ratio of the flash to background intensity (see Materials and Methods). Since the backgrounds cover a range of some 50,100 , the magnitude of photon flux generated by any contrast ratio also varies by $50,100 \times$ across the three backgrounds. From this vantage, the differences in the amplitude of the responses to a fixed contrast ratio as a function of background intensity are rather minor. On the other hand, it is well known, and Figure 9 also shows, that the kinetics of the responses of photoreceptors are modified by light adaptation. In sum, the results in Figure 9 may be about as close as cones can come to achieving perfect contrast constancy over an appreciable range of background illumination.

A quantitative view of the occurrence and breakdown of contrast constancy (for peak amplitude) is given in Figure 10 for results obtained from cone $A$. Plotted here is the flash intensity required to evoke a response of fixed amplitude as a function of the background intensity for both incremental (open symbols) and decremental flashes (solid symbols). The size of the fixed criterion response, as a fraction of $V_{\max }$, is shown by each curve. Contrast constancy for peak amplitude holds exactly wherever the slope of the curves is 1.0. For reference, the dashed line has unit slope and represents the case where there is no change between the intensity in the background and that in the flash (see Materials and Methods). The dashed line may also be described as the relationship that is expected to hold for perfect contrast constancy in the limit as the flash intensity asymptotically approaches the background intensity. Hence, the experi- 
Contrast Ratio (F/B)

10

3.16
1.58
0.316

0.10

50,100

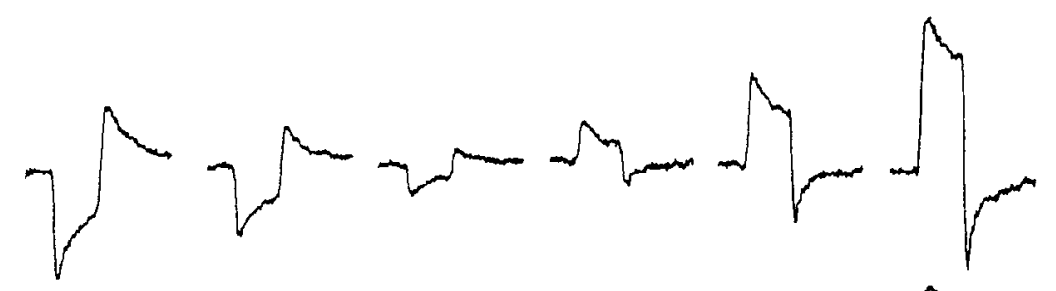

250

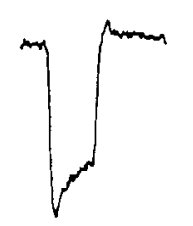<smiles>CCCCCCCCCCCCCCCCCCCCCCCC</smiles><smiles>CCCCCC</smiles><smiles>CCCCCCCCCCCCCCC</smiles>

1

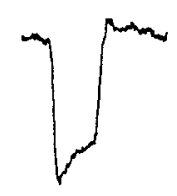
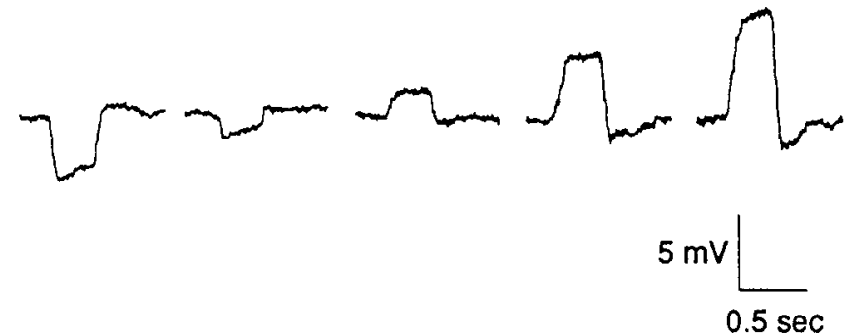

Figure 9. Approximate contrast constancy of the cone response over a $50,100 \times$ range of background illumination. The number to the left gives the relative intensity of the background illumination, where $1=-0.75 \log$ sigma (3.25 log photons $\left.\mathrm{sec}^{-1} \mu \mathrm{m}^{-2}\right), 250=$ $1.65 \mathrm{log}$ sigma $\left(5.65 \mathrm{log}\right.$ photons $\mathrm{sec}^{-1}$ $\left.\mu \mathrm{m}^{-2}\right)$, and $50,100=3.95 \log$ sigma (7.95 log photons $\sec ^{-1} \mu \mathrm{m}^{-2}$ ). The contrast ratio is given as the ratio of the flash intensity to the background intensity (see Materials and Methods). Selected records are from the cone of Figure 6.

mental measurements for decremental and incremental steps lie below and above the dashed line, respectively. Although rigure 10 shows that contrast constancy breaks down markedly at the extremes of background intensity and more so for larger critcria, the domain of approximate constancy is still rather remarkable, particularly when it is realized that, over the region where invariance holds for criterion responses of $30 \%$ and $40 \%$, the size of the corresponding steps are rather large-namely, about 0.75 and $1.0 \log$ unit, respectively.

Contrast symmetry, the special case in which equal contrast steps of opposite polarity evoke responses of equal absolute amplitude, is found in Figure 8 wherever the positive and negative segments of the curves remain symmetrical. In Figure 10, symmetry holds wherever the data for corresponding positive and negative criteria are oppositely but equally displaced from the dashed line. Although such symmetry breaks down markedly at the extremes of background intensity and for large criterion responses, Figure 10 shows that it holds reasonably well over a very large range of background intensity for a criterion response of $20 \% V_{\max }$. These responses are evoked by rather large steps in contrast, about $\pm 0.5 \log$ unit, that is, $3 \times$ and $1 / 3 \times$ for positive and negative contrast, respectively.

\section{Weber's law behavior}

The occurrence of contrast constancy in Figure 10 means that responses of relatively large amplitude conform to Weber's law

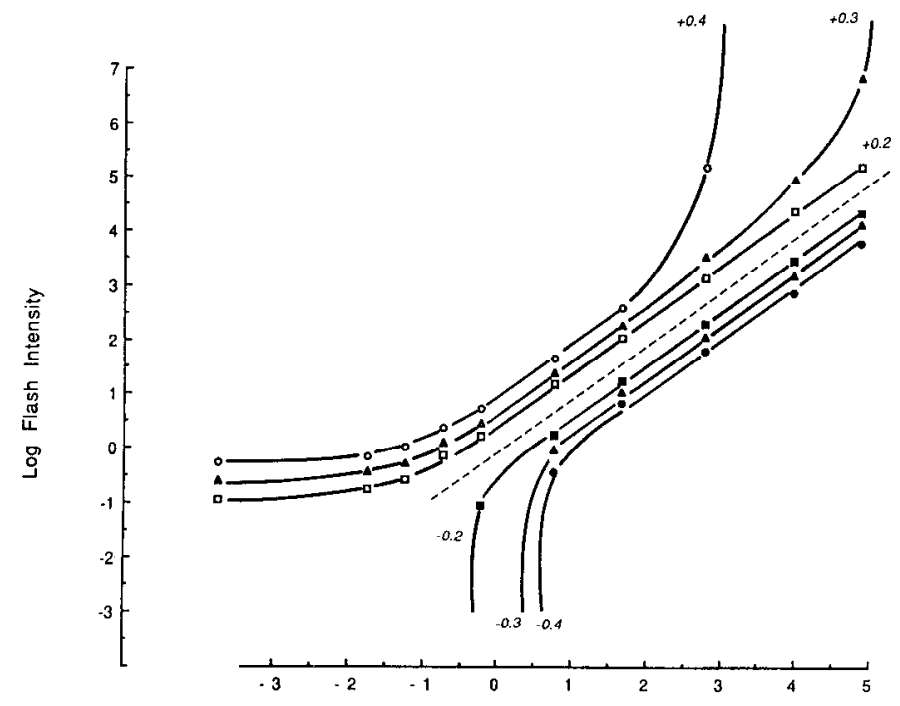

Log Background Intensity (re: sigma)

Figure 10. Flash intensity required to evoke a fixed criterion response as a function of the intensity of steady background illumination. The number by each curve indicates the criterion response as a fraction of the maximum response ( $V_{\text {max }}$ ) evoked in the dark, following the convention given for Figure 8 . Positive and negative criteria are for incremental and decremental responses, respectively. Dashed line represents the locus for flash intensity $=0$ (see Results). Smooth curves were drawn by eye. 


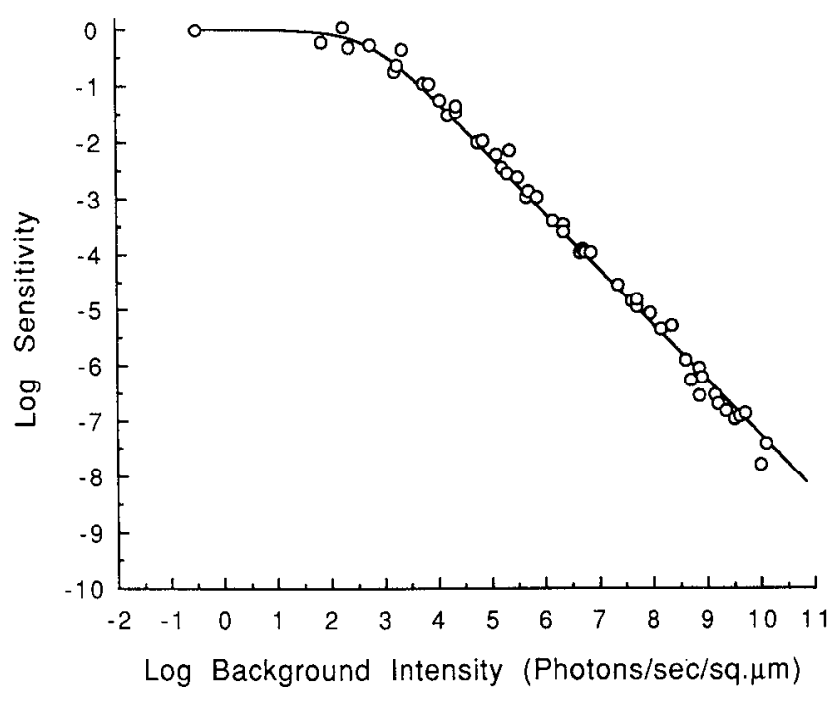

Figure 11. Step sensitivity for 15 cones as a function of the intensity of steady background illumination. The sensitivity is based on a criterion response of $5 \%$ of $V_{\max }$, the cone's maximum response in the fully darkadapted state. The point at the upper left gives the step sensitivity measured in the fully dark-adapted state. The data fall with a slope of about -1 over the upper 6.5-7 log units of background intensity and thus conform closely to Weber's law behavior. The equation for the smooth curve is given in Results (Eq. 6).

$(\Delta I / I=k)$ since whenever the data have unit slope, the relationship, $\Delta I / I=k$, is also satisfied. Thus, Weber's law holds rather well for criterion responses of $20 \% V_{\max }$ and over a smaller but still substantial range for even larger criterion responses. All the data in Figure 10 are for responses outside the linear range since, in plots of peak amplitude versus stimulus magnitude $(\Delta I)$, responses of $20 \% V_{\max }$ were clearly in the nonlinear domain. On the other hand, responses of $\leq 10 \%$ appeared to fall within the linear range, in agreement with past work on turtle cones (Baylor and Hodgkin, 1974; Normann and Anderton, 1983). Measurements of incremental sensitivity for a criterion response of $5 \%$ of $V_{\max }$ are shown in Figure 11 for data collected from a samplc of 15 cones. In this plot, Weber's law holds if sensitivity falls with -1 slope. In fact, this relationship holds closely over the upper $7 \log$ units of background illumination. The smooth curve which fits the full range of the data is given by the equation

$$
S_{L} / S_{D}=\left(1+I / I_{0}\right)^{-1},
$$

where $S_{L}$ and $S_{D}$ are the sensitivity to a step (400 msec) in the presence of background light and in the dark, respectively, $I$ is the intensity of the background light, and $I_{0}$ is the background intensity that reduces the step sensitivity by one-half (Baylor and Hodgkin, 1974; Matthews et al., 1990; Schnapf et al., 1990). The value for $I_{0}$ in Figure 11 is $2.70 \log$ photons $\sec ^{-1} \mu \mathrm{m}^{-2}$.

\section{Responses on very intense backgrounds}

Variability between cones in our sample was typically most apparent at relatively high background intensities (>7-8 log photons $\sec ^{-1} \mu \mathrm{m}^{-2}$ or, equivalently, about $3-4 \log$ sigma). Two apparently independent sources of variability were observed. (1) Variation in the total response range was seen. Although some variability in the range to both increments and decrements was observed, the greater variation was seen for decrements. The response to a full decrement (dark flash) sometimes re- mained relatively large (about $0.4-0.5$ ) on higher backgrounds, depolarizing nearly to the level of dark-adapted membrane potential, as in Figure 8. In other cones, however, the depolarizing response did not come close to the dark level and the response amplitude was correspondingly small, in the $0.20-0.25$ range. These small responses were also often sluggish. (2) In some cones, a decrease in the slope of the stimulus-response curve was seen for backgrounds at or above approximately $9 \log$ photons $\sec ^{-1} \mu \mathrm{m}^{-2}$ (approximately $5 \log$ sigma). Such decreases in slope imply a breakdown in strict Weber's law behavior. To examine this more closely, the data from all the cones of Figure 11 were analyzed by replotting the data as $\Delta I / I$ versus $\log I$. The Weber fraction, $\Delta I / I$, was about 0.30 and did not vary systematically over a range of background from about 3.5 to 9 $\log$ photons $\sec ^{-1} \mu \mathrm{m}^{-2}$. For about half the cones tested on backgrounds above about $9 \log$ photons $\sec ^{-1} \mu \mathrm{m}^{-2}$ the Weber fraction tended to increase somewhat, while others showed little change from that seen on weaker backgrounds.

\section{Discussion}

Weber's law and responses to contrast steps in cones

Intracellular recordings from cones have yielded a fair body of evidence for Weber's law behavior for small responses in various vertebrates over the years (Shaplcy and Enroth-Cugcll, 1984; Burkhardt and Gottesman, 1987; Dowling, 1987; Dawis, 1991), but that previously obtained from turtle cones (Normann and Anderton, 1983) stands out as the most precise and extensive, showing Weber's law over a change in sensitivity of about 3.5 log units. The present report now extends measurements on turtle cones to considerably more intense backgrounds and thereby shows that Weber's law behavior holds rather closely for small responses over some $7 \log$ units of change in cone sensitivity. By comparison, the largest range yet reported for human cone vision is about $5 \mathrm{log}$ units and is probably mediated by more than one cone type (Whittle, 1993). The photometric measurements described in Materials and Methods indicate that the most intense background level studied here (about $10 \mathrm{log}$ photons $\sec ^{-1} \mu \mathrm{m}^{-2}$ ) would correspond to a luminance of about $7.2 \log$ millilamberts ( $8.8 \mathrm{log}$ trolands), a luminance about 1000 times more intense than that of a white paper illuminated by sunlight at noon (Riggs, 1965). Thus, in nature and for small responses, Weber's law evidently rules the entire upper range of turtle vision. Although some cones may show some elevation in the Weber fraction at very intense levels, the elevation is rather unremarkable on the sort of logarithmic plots like Figure 11 that have commonly been used to demonstrate Weber's law behavior in vertebrate photoreceptors. Thus, even at the extremely high light levels used in the present work, there is yet no compelling evidence for saturation in turtle cones, in marked contrast to clear findings for saturation in rod photoreceptors (Fain, 1976; Baylor, 1987; Dowling, 1987).

The background intensity, $I_{0}$, that reduces the step sensitivity by one-half, was found to be $2.70 \log$ photons $\sec ^{-1} \mu \mathrm{m}^{-2}$ for the smooth curve in Figure 11. To compare our work with other reports on cones, however, it is necessary to determine $I_{0}$ for flash sensitivity rather than for step sensitivity. This was done in a sample of three cones and gave an average value of 3.06 $\log$ photons $\sec ^{-1} \mu \mathrm{m}^{-2}$. This is smaller than the value of 3.70 $\log$ photons $\sec ^{-1} \mu \mathrm{m}^{-2}$ estimated from one earlier report for cones in the eyecup (Normann and Anderton, 1983). On the other hand, Baylor and Hodgkin (1974) estimated $I_{0}$ at about 2000 photoisomerizations $\mathrm{sec}^{-1}$, whereas our finding of 3.06 
$\log$ photons $\sec ^{-1} \mu \mathrm{m}^{-2}$ at $632.8 \mathrm{~nm}$ is equivalent to about 16,000 photoisomerizations $\sec ^{-1}$. Values of about 3500 and 26,000 photoisomerizations $\mathrm{sec}^{-1}$, respectively, have been reported for isolated salamander cones (Matthews et al., 1990) and isolated monkey cones (Schnapf et al., 1990). Because the value for $I_{0}$ found for isolated monkey cones is about $10-100$ times greater than that found in human psychophysics or monkey ganglion cells (Purpura et al., 1990; Schnapf et al., 1990), postreceptor mechanisms are thought to contribute significantly to the overall reduction in visual sensitivity, at lcast at low background intensities. In the turtle, this issue is currently unsettled since the available psychophysical data (Granda and Sisson, 1989, their Fig. 3) suggest an $I_{0}$ value similar to that reported here for red-sensitive cones, whereas observations on cones and ganglion cells in the turtle retina (Baylor and Fettiplace, 1977) provide evidence for sensitivity reduction beyond the receptor level.

Although very small response criteria in the linear response range have typically been used to investigate Weber's law behavior in vertebrate cones, Figure 10 shows that Weber's law behavior is relatively robust and holds rather closely over 4-5 $\log$ units of background for relatively large response criteria of $\pm 20 \% V_{\max }$. These responses correspond to relatively large contrast steps $(\geq 3 \times)$ and their amplitudes are some $400 \times$ greater than the $5-12 \mu \mathrm{V}$ signals believed to mediate visual threshold in turtlc (Granda and Dvorak, 1977; Normann and Anderton, 1983). These results in the nonlinear, suprathreshold domain provide clear evidence for what has been called strong Weber's law behavior for cones (Dawis, 1991) or contrast constancy. In human vision, there is psychophysical evidence for contrast constancy as well as contrast symmetry over several decades of background illumination for contrasts up to at least $3 \times$ (Burkhardt et al., 1984; Walraven et al., 1990; Whittle, 1993). Figures 8-10 suggest that a substantial component of contrast constancy and symmetry may originate in cones.

\section{Changes in cones due to background illumination}

The changes in waveform associated with light adaptation (Fig. 6) resemble those described previously (Baylor and Hodgkin, 1974; Normann and Perlman, 1979). These changes might arise in the outer segment from changes in the kinetics of the underlying biochemical reactions of phototransduction (Baylor and Hodgkin, 1974; Baylor et al., 1979; Fain and Matthews, 1990; Matthews et al., 1990; Dawis, 1991) and/or from voltage-sensitive conductances in the inner segment (Normann and Perlman, 1979; Schnapf and McBurney, 1980; Barnes and Hille, 1989; Lasater and Witkovsky, 1991; Thoreson and Burkhardt, 1991; Lasater, 1992). It is well established that light adaptation leads to changes in temporal integration in cones (Baylor and Hodgkin, 1974; Tranchina et al., 1984; Daly and Normann, 1985). This factor would be expected to affect the slope of incremental sensitivity measurements when long flashes are used, as in the experiments of Figure 11. Although this question has not been investigated exhaustively, results obtained with either $20 \mathrm{msec}$ or $400 \mathrm{msec}$ flashes seemed to conform to Weber's law $(-1$ slope) about equally well over most of the background intensity range. Measurements of temporal integration in our preparations typically ranged from about $90 \mathrm{msec}$ when fully dark adapted to $30 \mathrm{msec}$ when fully light adapted, a smaller range than that reported previously (Baylor and Hodgkin, 1974; Daly and Normann, 1985). The change in temporal integration seemed largely confined to weak background intensities below
$+1 \log$ sigma. Empirically, Figure 11 shows that, whatever changes in temporal integration may be occurring, Weber's law holds as well or better for $400 \mathrm{msec}$ flashes as that reported previously for brief flashes.

We have found that the total range of the stimulus-response curve changes as a function of background illumination. Changes of this sort were not as striking in past work (Normann and Perlman, 1979), probably because the earlier measurements did not extend into the high-intensity realm investigated in the present report. Thus, our findings show that the effect of light adaptation cannot be simply described as a shift in a fixed template curve downward and rightward along the log intensity axis. We have also shown that the maximum response to a full decrement increases and then decreases as the background illumination is raised. Hence, there is an intermediate level of background illumination on which the range of response to negative contrast is maximal. This seems the consequence of three interacting factors. (1) The increase in the prevailing steady-state membrane potential over moderate levels of background illumination permits a larger range for the response. (2) A depolarizing overshoot further extends the response range on moderate backgrounds (Fig. 6C). (3) A reduction in the rate of the depolarization comes into play at very high intensities, thus reducing the response. This effect may involve recovery from bleaching since it begins to appear at background intensities of about 6-7 log photons $\sec ^{-1} \mu \mathrm{m}^{-2}$.

\section{Photopigment bleaching and mechanisms of light adaptation}

Our observations on time course, steady-state behavior, and action spectra (Figs. 3-5) support the conclusion that the transmission measurements reflect changes in the photopigment of red-sensitive cones. To our knowledge, these are the first measurements of bleaching of cone photopigments in situ, other than that obtained by the method of reflection densitometry in the human and monkey eye. At least three properties of the turtle eye seem fundamental for the success of the transmission method: (1) the opaque scleral tunic can be teased away, (2) the main body of the sclera is transparent, (3) the red-sensitive cones are by far the most numerous receptors in the retina. Consequently, our measuring beam of $632.8 \mathrm{~nm}$ can be highly absorbed by the parent pigment while being little influenced by absorption by other receptors or by photoproducts of bleaching that absorb in the short-wavelength region of the spectrum.

Our primary finding shows that the bleaching curve for turtle cones (Fig. 4) is well fitted by the equation previously applied to human cones (Rushton, 1972). The half-bleaching constant, $I_{h}$, of $5.5 \mathrm{log}$ photons $\mathrm{sec}^{-1} \mu \mathrm{m}^{-2}$ is surprisingly similar to the estimated value of $5.4 \mathrm{log}$ photons $\mathrm{sec}^{-1} \mu \mathrm{m}^{-2}$ for human and monkey cones, obtained by converting the previously reported value of about $4.3 \mathrm{log}$ trolands (Rushton and Henry, 1968; Hollins and Alpern, 1973; Valeton and van Norren, 1983) by factors appropriate for the human and monkey eye (Wyszecki and Stiles, 1982; Schnapf et al., 1990). When converted to photoisomerizations, the half-bleaching constant for monkey cones (Valeton and van Norren, 1983) and the present results for turtle cones are, respectively, about 5.40 and $6.35 \mathrm{log}$ photoisomerization $\sec ^{-1}$ per cone. It thus appears that turtle cones are somewhat more resistant to bleaching by prolonged background illumination. On various assumptions, Normann and Perlman (1979) estimated $I_{h}$ for turtle cones at about $5.95 \mathrm{log}$ photons $\mathrm{sec}^{-1} \mu \mathrm{m}^{-2}$, about 2.8 times higher than the actual measurements reported here. 

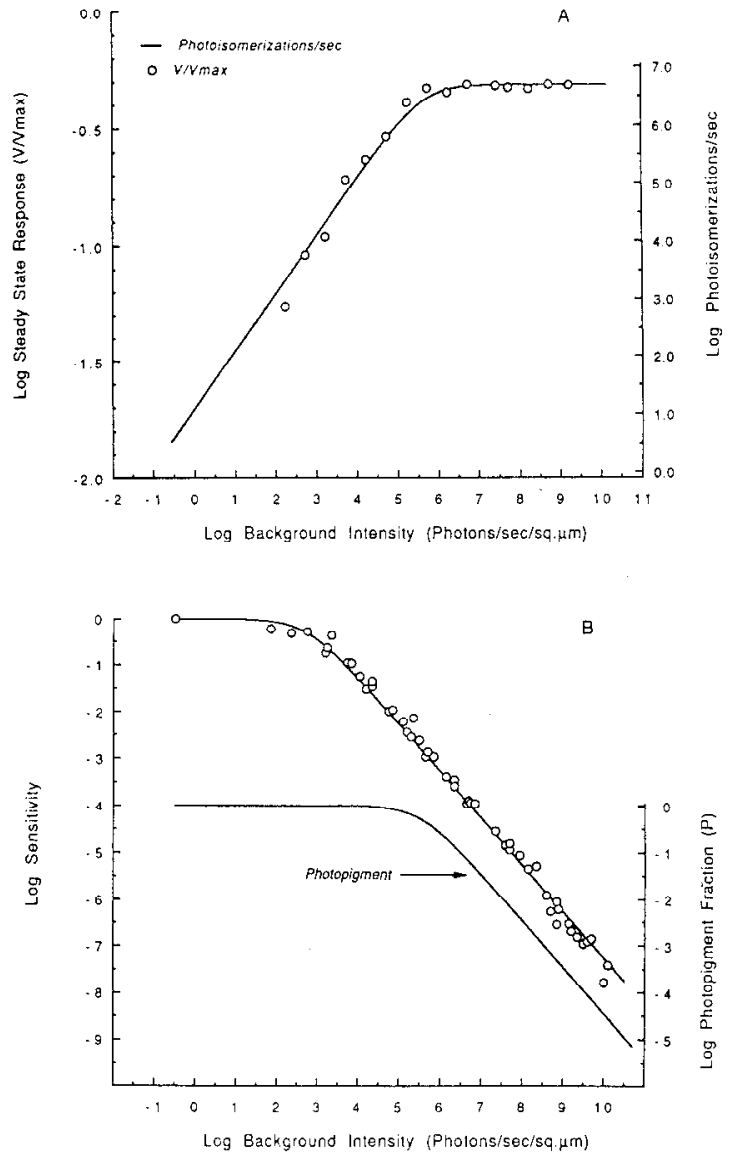

Figure 12. Comparison of aspects of light adaptation in red-sensitive cones and changes in photopigment as measured by light transmission. $A$, Maintained potential in the steady state (open circles, left ordinate) and rate of photoisomerization (smooth curve, right ordinate). $B$, Step sensitivity for results collected from 15 cones (open circles, left ordinate) and estimated value of $P$, the fraction of cone photopigment unbleached (right ordinate, lower smooth curve). The smooth curve drawn through the open circles for the step sensitivity is the same as that in Figure 11 with $I_{0}=2.70 \log$ photons $\mathrm{sec}^{-1} \mu \mathrm{m}^{-2}$. The smooth curve for the photopigment is based on the equation $P=I_{h} /\left(I_{h}+I\right)$, where $I_{h}$ is the average experimentally measured value, equivalent to $5.54 \log$ photons $\sec ^{-1} \mu \mathrm{m}^{-2}$ (see Results).

Quantitative comparisons of our measurements of photopigment bleaching and light adaptation are given in Figure 12. The smooth curve in $A$ shows the rate of photoisomerizations $\mathrm{sec}^{-1}$ per cone in the steady state, calculated from the product, $I \cdot f$. $q$, where $I$ is the number of photons $\sec ^{-1}$ incident upon the outer segment (Baylor and Fettiplace, 1975), $f$ is the fraction of incident photons absorbed, and $q=0.65$, the quantum efficiency. The fraction absorbed, $f$, was calculated from the modified relation (Fein and Szuts, 1982) $f=1-10^{-d p}$, where $d$, the optical density in the fully dark-adapted state, is taken as 0.195 (Baylor and Fettiplace, 1975), and $p$ is the proportion of pigment unbleached based on the fit of the bleaching equation $\left(I_{h}=5.54\right.$ $\log$ photons $\sec ^{-1} \mu \mathrm{m}^{-2}$ ) to our average transmission measurements. In Figure 12A, the ordinate for the photoisomerization rate has been positioned vertically and scaled down by a factor of about 4 relative to the left ordinate to bring the smooth curve in approximate register with the open circles. The open circles are the average steady-state measurements of Figure 7, here plotted logarithmically. The near coincidence of the two sets of results indicates that there is a power law relationship between the steady-state hyperpolarization and the photoisomerization rate. Linear regression analysis gave the best-fitting equation

$$
R_{s}=k \cdot \mathrm{Pi}^{0.268}
$$

where $R_{s}$ is the normalized steady-state response $\left(V / V_{\max }\right), \mathrm{Pi}$ is in photoisomerizations $/ \mathrm{sec}, k=0.0082$, and the best-fit index, $r^{2},=0.983$. The functional significance of this equation in relation to the complex cascade of reactions intervening between photoisomerization and membrane potential is unknown.

The present results show that the steady-state potential and the photoisomerization rate stabilize concurrently over the upper 4 decades of background illumination (approximately $10^{6}$ to $10^{10}$ photons $\sec ^{-1} \mu \mathrm{m}^{-2}$ ). The stabilization of the membrane potential thus seems explained by the stabilization of the photoisomerization rate and it appears that under steady-state conditions of light adaptation, about $5 \times 10^{6}$ photoisomerizations $\mathrm{sec}^{-1}$ lead to the closure of about half of the cone's light-sensitive channels. The stabilization of the photoisomerization rate is due, in turn, to the depletion of photopigment by bleaching. Over the range from about $10^{6}$ to $10^{10}$ photons $\mathrm{sec}^{-1} \mu \mathrm{m}^{-2}$, the increase in the number of incident photons due to raising the background intensity is exactly offset by the decrease in the photon catch due to photopigment depletion. Thus, the stabilization of the membrane potential at about $0.5 V_{\max }$ is the consequence of photopigment bleaching.

Figure $12 B$ shows the pigment bleaching curve in logarithmic coordinates along with the step sensitivity results from Figure 11 . The bleaching curve has been vertically positioned to highlight the region over which both curves decline in parallel with -1 slope. It is thus evident that over the range of background from about 6-6.5 to $10 \mathrm{log}$ photons $\mathrm{sec}^{-1} \mu \mathrm{m}^{-2}$, photopigment bleaching can account for the progressive reduction in cone sensitivity (Webcr's law); that is, as the background intensity is raised, the photopigment is proportionately depleted, so the intensity of the incremental test flash $(\Delta I)$ must be proportionately raised to generate a fixed response. The range of background intensity over which such proportional depletion of photopigment occurs (Fig. 12B) is virtually identical to that for the stabilization of the steady-state membrane potential (Fig. 12A).

It is sometimes suggested that depletion of photopigment may allow cones to adapt indefinitely and thus avoid saturation on even the most intense backgrounds. This will be true, however, only if the number of unbleached photopigment molecules is sufficient to accommodate the number of incoming photons in the test flash (Dartnall, 1969). This question was examined by calculating the number of photoisomerizations required to evoke a fixed response of $5 \% V_{\max }$ and comparing this to the number of available, unbleached molecules.

The photoisomerization rate for the incremental test flash $(\Delta I)$ in the presence of a given background was calculated from the product $\Delta I \cdot f \cdot q$. where $f$ and $q$ are as defined above and the test flash was assumed to produce negligible bleaching. This value was multiplied by the cone's integration time to give the number of photoisomerizations required to evoke the criterion response. From experimental results described above, integration times of 90 and 30 msec were taken for the fully dark-adapted and fully light-adapted states, respectively. It was found that the number of required photoisomerizations/flash was about 150 in the dark-adapted state and rose with background intensity to reach an asymptotic value of about 46,000 /flash for backgrounds above approximately $6.5 \mathrm{log}$ photons $\mathrm{sec}^{-1} \mu \mathrm{m}^{-2}$. The number of available, unbleached molecules was taken as $P \cdot 10^{8}$, 
where $P$ was laken from Equation 4 describing the smooth curve in Figure $12 B$, and $10^{8}$ is the estimated number of photopigment molecules in red-sensitive turtle cones (Hodgkin and O'Bryan, 1977). Over most of the range of background intensity, the number of available (unbleached) molecules substantially exceeded the number of photoisomerizations required to mediate the test flash response. However, for backgrounds of 9 and 10 $\log$ photons $\mathrm{sec}^{-1} \mu \mathrm{m}^{-2}$, respectively, the calculations indicated that about 35,000 and 3500 molecules remained unbleached, whereas 46,000 photoisomerizations/flash were required for the criterion response. Thus, at the most intense background investigated ( $10 \log$ photons $\sec ^{-1} \mu \mathrm{m}^{-2}$ ), it appears that about 10 times more molecules are required for the incremental response than are available. Nevertheless, a marked breakdown in Weber's law was not observed experimentally (Fig. 11). A ready explanation for this discrepancy is not at hand. Thus, the value of $10^{8}$ molecules/cone, although not measured directly, is consistent with accepted properties of visual photopigments and the dimensions of turtle cones (Hodgkin and O'Bryan, 1977) and is most unlikely to be some 10 times larger, as would be required to explain the present discrepancy. The assumption of negligible regeneration is compatible with the accepted view that regeneration rate should be very low when most of the photopigment is bleached (see, e.g., Rushton, 1972). In marked contrast, ultrafast regeneration on the order of 1500 molecules $/ \mathrm{msec}$ would seem necessary to boost the number of photoisomerizations/flash (30 $\mathrm{msec}$ integration time) to the approximately 46,000 required. Although the possibilities given above seem implausible and/or insufficient by themselves, it should be emphasized that very little is as yet known about the behavior of cone photopigment molecules in intact cones at the exceedingly high intensity levels investigated in the present report. Moreover, as discussed in Results, there is some suggestion that the cone may be coming near the end of its operating range for background jllumination in the region of $10 \mathrm{lng}$ photons $\mathrm{sec}^{-1}$ $\mu \mathrm{m}^{-2}$

In contrast to the 6.5-7 log units range reported here for cones in situ, recent reports on isolated cones show Weber's law behavior over 1.5-2.5 log units, above which largely irreversible sensitivity losses occur (Matthews et al., 1990; Schnapf et al., 1990). The limited ranges are probably due, at least in part, to a greatly depressed capacity for photopigment regeneration in isolated cones. When turtle cones are deprived of their normal association with the pigment epithelial cells in an isolated retina preparation, Weber's law behavior is observed over only about $2 \log$ units of sensitivity up to a background intensity of about $5.5 \mathrm{log}$ photons $\mathrm{sec}^{-1} \mu \mathrm{m}^{-2}$, above which a largely irreversible saturation occurs (Normann and Perlman, 1990). In stark contrast, Figure $12 B$ shows that a background impingement of 5.5 log photons $\sec ^{-1} \mu \mathrm{m}^{-2}$ is easily accommodated by cones in situ, and that normal light adaptation will prevail over some four additional decades of background light. Thus, the pigment epithelium and perhaps additional features of the intact retina seem crucial for preserving the immense range of light adaptation reported here for cones in situ.

Figure 12B shows that there is a large range of background illumination from about 2.5 to $5.5 \log$ photons $\mathrm{sec}^{-1} \mu \mathrm{m}^{-2}$ where light adaptation in red-sensitive cones of the turtle does not depend on photopigment depletion, confirming the inference of Normann and Perlman (1979). Recent experiments in salamander cones and rods strongly suggest that changes in intracellular calcium may regulate the receptor's sensitivity over the lower 2-3 log units of the Weber's law range (Fain et al., 1989; Fain and Matthews, 1990; Matthews et al., 1990). This mechanism might be the primary or sole basis for light adaptation in turtle cones at the low-to-intermediate light levels identified in the present report where photopigment depletion is inconsequential. On the other hand, Dawis (1991) has described a comprehensive quantitative model in which mechanisms involving calcium-independent aspects of the cGMP metabolic cycle could both modulate the sensitivity and waveform of the cone response while also giving rise to strong Weber's law behavior in cones.

\section{Appendix}

The purpose of this section is to present a mathematical analysis of the expected contribution of all classes of photoreceptors of the turtle retina to the photodiode measurements reported in the body of the present report.

The photiodide signal, $s$, arising from illumination of a single photoreceptor is assumed to be proportional to the intensity of the measuring light, $I_{m}$, and the fraction of light transmitted, $T$ :

$$
s=k \cdot I_{m} \cdot T
$$

where $k$ is a constant depending on the sensitivity of the photodiode and the efficiency of the collecting optics. Equation 8 may be rewritten in terms of $f_{a}$, the fraction of light absorbed by the photopigment:

$$
s=k \cdot I_{m} \cdot\left(1-f_{a}\right)
$$

The quantity $f_{a}$ is computed (Fein and Stutz, 1982)

$$
f_{a}=1-10^{-D \cdot A_{w} \cdot r} \text {, }
$$

where $D$ is the optical density of the photopigment at the wavelength of maximum absorption when the photopigment is fully regenerated, $A_{w}$ is the absorption coefficient at the wavelength of the measuring light $(632.8 \mathrm{~nm})$, and $P$ is the fraction of pigment unbleached.

The quantity $I_{m}$ and the constant $k$ in Equation 8 can be neglected since both are held constant throughout the experiment and absolute values are not important for the present purposes. Combining Equations 9 and 10 then gives

$$
s=10^{-D \cdot A_{w} \cdot P .}
$$

The value of $D$ is taken as 0.195 for red-sensitive cones (Baylor and Fettiplace, 1975) and is assumed to also apply for the greensensitive cones. In the micrographs of Kolb and Jones (1982), the outer segments of rods are about twice as long as that of the red-sensitive cones, and so the photopigment density of the rods is taken as 0.39 , that is, twice that of the cones. For a measuring light of $632.8 \mathrm{~nm}, A_{w}$ is taken as $1.0,0.04$, and 0.04 , respectively, for the photopigment of the red-sensitive cones, green-sensitive cones, and rods, based on the nomogram for A2 photopigments having maximum absorption at about 625 and $520 \mathrm{~nm}$ (Ebrey and Honig, 1977). A value of 0.001 is estimated for $A_{w}$ for the blue-sensitive cones, based on the action spectrum measurements of Baylor and Hodgkin (1973). This value and the relative incidence of the blue-sensitive cones-about $5 \%$ of the cone population (Ohtsuka, 1985)-are so low that the blue-sensitive cones can be neglected in the analysis that follows.

The fraction of pigment unbleached, $P$, is calculated from the steady-state bleaching equation (Rushton, 1972);

$$
P=I_{h} /\left(I_{h}+I\right)
$$


where $I$ is the intensity of the steady adapting light, and $I_{h}$ is the half-bleaching constant. It is assumed that the bleaching efficiencies, and thus the half-bleaching constants, $I_{h}$, of the photopigment of both cone types and the rods are equal when the photopigment is illuminated at the optimum wavelength. However, in the present experiments, the adapting light is fixed at $630 \mathrm{~nm}$. Thus, if $I_{h}$ is the bleaching constant for red-sensitive cones, that for green-sensitive cones and the rods must be scaled up by $1 / A_{w}$, that is, a factor of 25 , since $A_{w}$ at $630 \mathrm{~nm}=0.04$ for both classes of receptor as notcd above.

The resultant photodiode signal, $S$, arising from all photoreceptors illuminated by the measuring light is taken as the sum of the weighted signal arising from each class of receptor:

$$
S-\left(k_{r} \cdot S_{r}\right)+\left(k_{g} \cdot S_{g}\right)+\left(k_{\text {rod }} \cdot S_{\text {rod }}\right),
$$

where $k_{r}, k_{g}$, and $k_{\text {rod }}$ are weighting coefficients determined by the product of the relative number and the cross-sectional area of the photoreceptors illuminated. For convenience, the weighting coefficient for the red-sensitive cones is set to $1.0 ; k_{g}$ is then calculated as 0.20 , based on the findings that the relative incidence of the green-sensitive cones is about 0.25 (Ohtsuka, 1985) and the cross-sectional area of their outer segments is about 0.78 that of the red-sensitive cones (Baylor and Fettiplace, 1975). The value of $k_{\text {rod }}$ is calculated as 0.20 , based on a 0.05 incidence of rods relative to that of the red-sensitive cones (Ohtsuka, 1985) and an estimate from the micrographs of Kolb and Jones (1982), that the cross-sectional area of rods is about four times that of red-sensitive cones. Substituting Equation 11 into 13, and incorporating all constants given above, yields

$$
S=10^{-0.195 P_{r}}+0.20\left(10^{-0.008 P_{v}}\right)+0.20\left(10^{-0.015 P_{\mathrm{rod}}}\right) .
$$

When the retina is fully light adapted and all the photopigment depleted $\left(P_{r}=P_{g}=P_{\text {rod }}-0\right)$, calculations from Equation 14 show that the measured photodiode signal, $S$, will be increased by about 1.4 times above that measured in the fully dark-adapted state $\left(P_{r}=P_{g}=P_{\text {rod }}=1.0\right)$. However, about $97 \%$ of this change will be due to light passing through the red-sensitive cones and only $3 \%$ to light passing through the green-sensitive cones and rods. The small contribution of the latter receptors is largely due to the relatively long wavelength $(632.8 \mathrm{~nm})$ of the measuring light. Their photopigment is nearly transparent at $632.8 \mathrm{~nm}$ and thus transmits about $96 \%$ of the incident light under fully dark-adapted conditions. Hence, the transmission and relative contribution to the photodiode signal by these receptors can only increase by about $4 \%$, at most, when they are fully light adapted and all their photopigment is depleted. Thus, it seems evident that most of the change in the experimentally measured photodiode signal should be due to the red-sensitive cones. This issue was examincd quantitativcly by using Equation 14 to calculate the expected photodiode signal as a function of the intensity of steady background lights. The calculated signals were then used to calculate the expected values of $R, B$ and $P$, as defined by Equations 1 and 2 in Materials and Methods. It was found that the signals from the green-sensitive cones and rods had minor effects on the bleaching curve, distorting the shape slightly and increasing the apparent value of $I_{h}$ by about $0.10 \mathrm{log}$ unit. In particular, if $I_{h}$ for the red-sensitive cone photopigment was taken as $\log 5.43$ photons $\sec ^{-1} \mu \mathrm{m}^{-2}$, calculations from Equation 14 predicted that the experimentally measured value would be increased to 5.54 photons $\sec ^{-1} \mu \mathrm{m}^{-2}$. The latter value is, in fact, the average value measured experimentally (see Results). Thus, $I_{h}$ may be overestimated by about
$0.11 \log$ unit in our results. However, the difference in question is within the experimental error ( $\mathrm{SD}= \pm 0.13 \mathrm{log}$ unit) of our measurements (see Results), so no corrections based on Equation 14 were made.

Other calculations showed that in the action spectrum measurements of Figure 5, the measured value of $I_{h}$ for a $530 \mathrm{~nm}$ adapting light would be overestimated by about $0.16 \log$ unit due to influence of the green-sensitive cones and the rods. Since $I_{h}$ for a $630 \mathrm{~nm}$ adapting light is raised $0.11 \mathrm{log}$ unit (see above), the distortion in the action spectrum at 530 versus $630 \mathrm{~nm}$ amounts to about $0.05 \mathrm{log}$ unit relative to the difference expected for measurements arising purely from the red-sensitive cones. Thus, the distortion of the action spectrum measurements will be quite small. This is largely because both the green-sensitive cones and rods absorb very little at the wavelength $(632.8 \mathrm{~nm})$ of the measuring light (see above).

For convenience, the contribution of "stray light," that is, the component of the signal generated by the fraction of the measuring light that does not pass through the photoreceptors, has been ignored in the above analysis. It would amount to an added constant in the right side of Equation 14. However, as discussed in Materials and Methods in connection with Equation 3, the relative effect of stray light is factored out by the calculations in Equation 2, since $B$ is based on changes in ratios. Thus, the presence of stray light will not alter the conclusions reached above. The results of the present analysis strongly support the conclusion that the changes in transmission measured in the present paper arise almost exclusively from the red-sensitive cones, even under extreme conditions where the contribution of other receptors is maximized. It is therefore likely that the results in Figure 2 give a relatively uncontaminated view of the kinetics of the photopigment of the red-sensitive cones.

\section{References}

Alpern M (1971) Rhodopsin kinetics in the human eye. J Physiol (Lond) 217:447-471.

Baker H, Watson AJ, Coile CD (1991) Experimental stay light in retinal densitometry. Vis Neurosci 6:615-620.

Barnes S, Hille B (1989) Ionic channels of the inner segment of tiger salamander cone photoreceptors. J Gen Physiol 94:719-743.

Baylor DA (1987) Photoreceptor signals and vision. Invest Ophthalmol Vis Sci 28:34-49.

Baylor DA, Fettiplace R (1975) Light path and photon capture in turtle photoreceptors. J Physiol (Lond) 248:433-464.

Baylor DA, Fettiplace R (1977) Transmission from photoreceptors to ganglion cells in the turtle retina. J Physiol (Lond) 242:729-758.

Baylor DA, Hodgkin AL (1973) Detection and resolution of visual stimuli by turtle photoreceptors. J Physiol (Lond) 234:163-168.

Baylor DA, Hodgkin AL (1974) Changes in time scale and sensitivity in turtle photoreceptors. J Physiol (Lond) 242:729-753.

Baylor DA, Lamb TD, Yau KW (1979) The membrane current of single rod outer segments. J Physiol (Lond) 288:589-611.

Bonds AB, MacLeod DTA (1974) The bleaching and regeneration of rhodopsin in the cat. J Physiol (Lond) 242:237-253.

Boynton RM, Whitten DN (1970) Visual adaptation in monkey cones: recordings of late receptor potentials. Science 170:1423-1426.

Burkhardt DA, Gottesman J (1987) Light adaptation and responses to contrast flashes in cones of the walleye retina. Vision Res 27:14091420 .

Burkhardt DA, Gottesman J, Kersten D, Legge GE (1984) Symmetry and constancy in the perception of negative and positive luminance contrast. J Opt Soc Am Al:309-316.

Burkhardt DA, Gottesman J, Thoreson WB (1988) Prolonged depolarization in turtle cones evoked by current injection and stimulation of the receptive field surround. J Physiol (Lond) 407:329-348.

Burkhardt DA, Gottesman J, Thoreson WB (1989) An eyecup slice preparation for intracellular recording in vertebrate retinas. $J$ Neurosci Methods 28:179-187. 
Coble JR, Rushton WAH (1971) Stiles-Crawford effect and the bleaching of cone pigments. J Physiol (Lond) 217:231-242.

Copenhagen DR, Gree DG (1987) Spatial spread of adaptation within the cone network of turtle retina. J Physiol (Lond) 393:763-776.

Daly SJ, Normann RA (1985) Temporal information processing in cones: effects of light adaptation on temporal summation and modulation. Vision Res 25:1197-1206.

Dartnall HJA (1962) Investigation of visual pigments. In: The eye, Vol 2 (Davson H, ed), pp 235-256. New York: Academic.

Dartnall HJA (1969) The photochemical approach to visual problems. In: The retina: morphology, function and clinical characteristics (Straatsma BR, Hall MO, Allen RA, Crescitelli F, eds). Berkeley, CA: University of California.

Dawis SM (1978) A model for light adaptation: producing Weber's law with bleaching-type kinetics. Biol Cybern 30:187-193.

Dawis SM (1991) A molecular basis for Weber's law. Vis Neurosci 7:285-320.

Dowling JE (1987) The retina: an approachable part of the brain. Cambridge, MA: Belknap.

Ebrey TG, Honig B (1977) New wavelength dependent visual pigment nomograms. Vision Res 17:147-151.

Fain GL (1976) Sensitivity of toad rods: dependence on wavelength and background illumination. J Physiol (Lond) 261:71-101.

Fain GL, Matthews HR (1990) Calcium and the mechanism of light adaptation in vertebrate photoreceptors. Trends Neurosci 13:378384.

Fain GL, Lamb TD, Matthews HR, Murphy RWL (1989) Cytoplasmic calcium as the messenger for light adaptation in salamander rods. $J$ Physiol (Lond) 416:215-243.

Fein A, Szuts ET (1982) Photoreceptors: their role in vision. Cambridge: Cambridge UP.

Geisler WS (1978) The effects of photopigment depletion on brightness and threshold. Vision Res 18:269-278.

Granda AM, Dvorak CA (1977) Vision in turtles. In: Handbook of sensory physiology, Vol II/5, The visual system of vertebrates (Crescitelli F, ed), pp 451-495. New York: Springer.

Granda AM, Sisson DF (1989) Psychophysically derived visual mechanisms in turtle. I. Spectral properties. Vision Res 29:93-105.

Hecht S (1937) Rods, cones and the chemical basis of vision. Physiol Rev 17:239-290.

Hodgkin AL, O'Bryan PM (1977) Internal recording of the early receptor potential in turtle cones. J Physiol (Lond) 267:737-766.

Hollins M, Alpern M (1973) Dark adaptation and visual pigment regeneration in human cones. J Gen Physiol 62:430-447.

Hood DC, Birch DG (1993) Human cone activity: the leading edge of the a-wave and models of receptor activity. Vis Neurosci 10:857872 .

Kolb H, Jones J (1982) Light and electron microscopy of the photoreceptors in the retina of the red-eared slider, Pseudemys scripta elegans. J Comp Neurol 209:331-338.

Lasater EM (1992) Membrane properties of distal retinal neurons. In: Progress in retinal research, Vol 11 (Osborne NN, Chader CJ, eds), pp 215-246. Oxford: Pergamon.

Lasater EM, Witkovsky P (1991) The calcium current of turtle cone photoreceptor axon terminals. Neurosci Res [Suppl] 11 5:S165-S173.

Matthews HR, Fain GL, Murphy RLW, Lamb TD (1990) Light adaptation in cone photoreceptors of the salamander: a role for cytoplasmic calcium. J Physiol (Lond) 420:447-469.

Normann RA, Anderton PJ (1983) The incremental sensitivity curve of turtle cone photoreceptors. Vision Res 23:1731-1733.
Normann RA, Perlman I (1979) The effects of background illumination on the photoresponses of red and green cones. J Physiol (Lond) 286:491-507

Normann RA, Pcrlman I (1990) Background and blcaching adaptation in luminosity type horizontal cells in the isolated turtle retina. J Physiol (Lond) 421:321-341.

Ohtsuka $\mathrm{T}$ (1985) Relation of spectral types to oil droplets in cones of turtle retina. Science 229:874-877.

Piccolino M, Gerschenfeld HM (1980) Characteristics and ionic processes involved in feedback spikes of turtle cones. Proc $\mathrm{R}$ Soc Lond [Biol] 206:439-463.

Pulvinage V, Green DG (1990) Square root intensity coding in turtle cones: physiological mechanisms. Vision Res 30:683-691.

Purpura K, Tranchina D, Kaplan E, Shapley RM (1990) Light adaptation in the primate retina: analysis of changes in gain and $\mathrm{dy}-$ namics of monkey retinal ganglion cells. Vis Neurosci 4:75-93.

Riggs LA (1965) Light as a stimulus for vision. In: Vision and visual perception (Graham CH, eds), pp 1-38. New York: Wilcy.

Ripps H, Weale RA (1965) Analysis of foveal densitometry. Nature 205:52-56.

Rushton WAH (1972) Visual pigments in man. In: Handbook of sensory physiology, Vol 7/1, The photochemistry of vision (Dartnall HJA, ed), pp 365-394. Berlin: Springer.

Rushton WA, Henry GH (1968) Bleaching and regeneration of cone pigments in man. Vision Res 8:617-631.

Schnapf JL, McBurney RN (1980) Light-induced changes in membrane current in cone outer segments of tiger salamander and turtle. Nature 287:239-241.

Schnapf JI., Nunn RJ, Meister M, Baylor DA (1990) Visual transduction in cones of the monkey Macaca fascicularis. J Physiol (Lond) 427:681-713.

Shapley R, Enroth-Cugell C (1984) Visual adaptation and retinal gain control. In: Progress in retinal research, Vol 3 (Osborne NN, Chader CT, eds), pp 263-346. Oxford: Pergamon.

Smith VC, Pokomy JL, van Norren D (1983) Densitometric measurement of human cone photopigment kinetics. Vision Res 23:517524.

Thoreson WB, Burkhardt DA (1991) Ionic influences on the prolonged depolarization of turtle cones in situ. J Neurophysiol 65:96-110.

Tranchina D, Gordon J, Shapley RM (1984) Retinal light adaptationevidence for a feedback mechanism. Nature 310:314-316.

Valeton JM, van Norren D (1983) Light adaptation of primate cones: an analysis based on extracellular data. Vision Res 23:1539-1547

van Norren D, van der Kraats J (1981) A continuously recording retinal densitometer. Vision Res 21:897-906.

Walls GL (1963) The vertebrate eyc and its adaptive radiation. New York: Hafner.

Walraven J, Enroth-Cugell C, Hood DC, MacLeod DIA, Schnapf JL (1990) The control of visual sensitivity: receptoral and postreceptoral processes. In: Visual perception: the neurophysiological foundations (Spillman L, Werner JS, eds), pp 53-102. New York: Academic.

Whittle P (1993) The psychophysics of contrast-brightness. In: Lightness, brightness and transparency (Gilchrist A, ed), in press. Hillsdale, NJ: Erlbaum.

Witkovsky P, Shi XP (1990) Slow light and dark adaptation of horizontal cells in the Xenopus retina: a role for endogenous dopamine. Vis Neurosci 5:405-413.

Wyszecki G, Stiles WS (1982) Color science. New York: Wiley. 